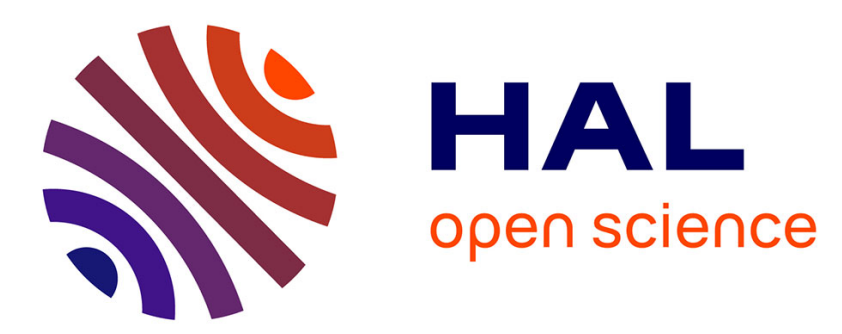

\title{
ADAM22 and ADAM23 modulate the targeting of the Kv1 channel-associated protein LGI1 to the axon initial segment
}

Bruno Hivert, Laurè Ne Marien, Komlanvi Nassirou Agbam, Catherine Faivre-Sarrailh

\section{To cite this version:}

Bruno Hivert, Laurè Ne Marien, Komlanvi Nassirou Agbam, Catherine Faivre-Sarrailh. ADAM22 and ADAM23 modulate the targeting of the Kv1 channel-associated protein LGI1 to the axon initial segment. Journal of Cell Science, 2019, 132 (2), pp.jcs219774. 10.1242/jcs.219774 . hal-02061637

\section{HAL Id: hal-02061637 \\ https://hal-amu.archives-ouvertes.fr/hal-02061637}

Submitted on 8 Mar 2019

HAL is a multi-disciplinary open access archive for the deposit and dissemination of scientific research documents, whether they are published or not. The documents may come from teaching and research institutions in France or abroad, or from public or private research centers.
L'archive ouverte pluridisciplinaire HAL, est destinée au dépôt et à la diffusion de documents scientifiques de niveau recherche, publiés ou non, émanant des établissements d'enseignement et de recherche français ou étrangers, des laboratoires publics ou privés. 


\title{
ADAM22 and ADAM23 modulate the targeting of the Kv1 channel-associated protein LGI1 to the axon initial segment
}

\author{
Bruno Hivert ${ }^{\star}$, Laurène Marien, Komlan Nassirou Agbam and Catherine Faivre-Sarrailh ${ }^{\ddagger}$
}

\begin{abstract}
The distribution of the voltage-gated $\mathrm{Kv} 1 \mathrm{~K}^{+}$channels at the axon initial segment (AIS) influences neuronal intrinsic excitability. The Kv1.1 and Kv1.2 (also known as KCNA1 and KCNA2, respectively) subunits are associated with cell adhesion molecules (CAMs), including Caspr2 (also known as CNTNAP2) and LGI1, which are implicated in autoimmune and genetic neurological diseases with seizures. In particular, mutations in the LGI1 gene cause autosomal dominant lateral temporal lobe epilepsy (ADLTE). Here, by using rat hippocampal neurons in culture, we showed that LGI1 is recruited to the AIS where it colocalizes with ADAM22 and Kv1 channels. Strikingly, the missense mutations S473L and R474Q of LGI1 identified in ADLTE prevent its association with ADAM22 and enrichment at the AIS. Moreover, we observed that ADAM22 and ADAM23 modulate the trafficking of LGI1, and promote its ER export and expression at the overall neuronal cell surface. Live-cell imaging indicated that LGI1 is co-transported in axonal vesicles with ADAM22 and ADAM23. Finally, we showed that ADAM22 and ADAM23 also associate with Caspr2 and TAG-1 (also known as CNTN2) to be selectively targeted to different axonal sub-regions. Hence, the combinatorial expression of Kv1-associated CAMs may be critical to tune intrinsic excitability in physiological and epileptogenic contexts.
\end{abstract}

KEY WORDS: Hippocampal neurons, Axonal transport, Epilepsy, ADLTE, $\mathrm{K}^{+}$channels

\section{INTRODUCTION}

The axon initial segment (AIS) is a unique compartment close to the cell body that is highly enriched in voltage-gated $\mathrm{Na}^{+}$channels and where the action potentials are initiated. The $\mathrm{Na}^{+}$channels are clustered at that site by a scaffold of ankyrinG (also known as ANK3) connected to spectrin and actin (Zhang and Rasband, 2016). The voltage-gated $\mathrm{Kv} 1 \mathrm{~K}^{+}$channels are likewise concentrated at the AIS, where they contribute to the control of neuronal excitability (Kole and Stuart, 2012; Rasband, 2010; Vacher and Trimmer, 2012). Kv1 channels co-purify with several cell adhesion molecules (CAMs) including Caspr2 (also known as CNTNAP2) and leucinerich glioma inactivated 1 (LGI1), which may influence their positioning within the distinct axonal sub-regions. The importance of these CAMs in neuronal function is reflected by their implicated role in both genetic and autoimmune diseases associated with

\footnotetext{
INMED, Aix Marseille Université, INSERM UMR1249, F-13273 Marseille, France. *Present address: Aix Marseille Université, CNRS UMR7289, Institut de Neurosciences de la Timone, F-13385 Marseille, France.

‡Author for correspondence (catherine.sarrailh@univ-amu.fr)

(D) B.H., 0000-0002-3371-6123; L.M., 0000-0003-1445-2940; K.N.A., 0000-00033045-6853; C.F., 0000-0002-1718-0533
}

Received 2 May 2018; Accepted 19 December 2018 hyperexcitability and epilepsy (Kegel et al., 2012; Lai et al., 2010; Muona et al., 2016; Ohkawa et al., 2013; Rodenas-Cuadrado et al., 2014). Antibody-mediated limbic encephalitis, which was first associated with Kv1 channels, has been mainly attributed to autoantibody binding to Caspr2 or LGI1 (Irani et al., 2010; Lancaster et al., 2011). Caspr2 is associated with TAG-1 (also known as CNTN2) at the juxtaparanodes of myelinated axons, where it mediates axo-glial contacts and induces the clustering of Kv1 channels to control the internodal resting potential (Poliak et al., 2003; Traka et al., 2003). In addition to juxtaparanodes, Caspr2 and TAG-1 are concentrated at the AIS of cortical and motor neurons, but they are not required for the recruitment of Kv1 channels at that site (Duflocq et al., 2011; Inda et al., 2006; Ogawa et al., 2008). In cultured hippocampal neurons, Kv1 channels are enriched at the AIS associated with TAG-1, whereas Caspr2 is targeted all along the axon (Pinatel et al., 2017). Other membrane proteins interacting with Kv1 channels may be localized at the AIS, including ADAM22, which is recruited at the AIS of cultured hippocampal neurons with PSD93 (also known as DLG2) (Ogawa et al., 2010). However, the precise mechanisms implicated in the recruitment of the Kv1 complex at the AIS are still elusive (Rasband, 2010). Actually, the polarized distribution of ion channels and CAMs at the somato-dendritic or axonal compartments may depend on several cellular mechanisms, including their sorting in distinct transport vesicles associated with specific kinesin motors, selective internalisation by endocytosis and diffusion/trapping in the cell membrane (Lasiecka et al., 2009). In particular, the AIS is a zone of restricted diffusion for the lateral mobility of transmembrane proteins, which are anchored to the ankyrin-spectrin-actin cytoskeleton. We previously reported that the Kv1-associated molecules TAG-1 and Caspr2 are co-transported in axonal vesicles whereas they are differently distributed along the axon (Bel et al., 2009; Pinatel et al., 2017). In the present study, we examined the role of ADAM proteins in the vesicular transport of distinct Kv1-associated CAMs, including TAG-1, Caspr2 and LGI1 and whether their interplay contributes to their targeting at the AIS.

LGI1 is a secreted glycoprotein consisting of leucine-rich and epitempin (EPTP) repeats that has been implicated in proteinprotein interactions at the synapse, but was not described at the AIS until recently (Seagar et al., 2017). LGI1 interacts, via its EPTP repeats, with members of the ADAM family, including ADAM11, ADAM22 and ADAM23 (Fukata et al., 2006; Owuor et al., 2009; Sagane et al., 2008). LGI1 has been proposed to form a transynaptic complex with ADAM22 and ADAM23 controlling synaptic strength at excitatory synapses by regulating PSD95 (also known as DLG4) incorporation (Fukata et al., 2010; Lovero et al., 2015). LGI1 at the pre-synaptic terminals has been reported to act as a negative modulator of glutamate release, an effect which could be linked with pre-synaptic Kv1 channels (Boillot et al., 2016). In patients with autoimmune encephalitis, anti-LGI1 antibodies may disrupt its interaction with ADAM proteins (Ohkawa et al., 2013). 
Furthermore, LGI1 is a monogenic human epilepsy-related gene mutated in autosomal dominant lateral temporal lobe epilepsy (ADLTE) (Gu et al., 2002; Kalachikov et al., 2002; Kegel et al., 2012; Morante-Redolat et al., 2002). LGI1 needs to be glycosylated in order to be secreted, and most ADLTE mutations inhibit LGI1 secretion by preventing its proper folding. Interestingly, some mutations do not inhibit secretion but were found to impair interactions with ADAM22 and ADAM23 (Dazzo et al., 2016; Yokoi et al., 2015).

Our present data indicate that LGI1 is enriched at the AIS where it colocalizes with ADAM22 and Kv1 channels in cultured hippocampal neurons. The ADAM proteins modulate the vesicular trafficking of LGI1, and promote its ER export and axonal transport leading to increased expression at the overall cell surface. In addition, we show that ADAM22 is critical for the enrichment of LGI1 at the AIS. Importantly, the secreted missense mutants LGI1 ${ }^{\text {S473L }}$ and LGI1 ${ }^{\text {R474Q }}$ identified in ADLTE display altered association with ADAM22 and are not properly targeted to the AIS. These mutations may induce perturbation of the LGI1 function in tuning intrinsic excitability, thus contributing to epileptogenesis.

\section{RESULTS}

LGI1 is targeted at the AIS of cultured hippocampal neurons

The Kv1 channels are known to associate with several membrane proteins, including ADAM22, ADAM23 and LGI1 at the presynaptic terminals. LGI1 was recently reported to be enriched at the AIS of hippocampal CA3 neurons in immunofluorescence staining studies on brain sections (Seagar et al., 2017). Here, we examined whether these CAMs may also interplay with the Kv1 complex at the AIS of hippocampal neurons in culture. In hippocampal neurons at DIV8 (day in vitro 8), we showed that LGI1 was faintly expressed at the AIS surface by live immunostaining with an anti-LGI1 monoclonal antibody $(\mathrm{mAb})$ (Fig. 1A, green), and colocalized with the AIS marker ankyrinG (Fig. 1A, blue). In contrast, at DIV21 when the synaptic network is established, LGI1 was present as small clusters on the somatodendritic compartment (Fig. 1B), and colocalized with the synaptic marker synaptobrevin (Fig. 1E, red). We observed that ADAM22 (Fig. 1C), but not ADAM23 (Fig. 1D), was enriched at the AIS by performing immunostaining after fixation and permeabilization, as described by Ogawa et al. (2010).

We next analyzed the subcellular distribution of LGI1-GFP transfected in hippocampal neurons. We observed that LGI1-GFP was highly restricted at the AIS surface when transfected at DIV7 and visualized at DIV8 using live immunostaining with anti-GFP antibody (Fig. 1F, red). The direct fluorescence of intracellular LGI1-GFP was faintly detected (Fig. 1F, green) and we decided for the next series of experiments to perform live immunostaining for GFP using Alexa Fluor 488 (green)-conjugated secondary antibodies. Surface LGI1-GFP (green) strongly colocalized with endogenous ADAM22 enriched at the AIS (blue), suggesting that the two proteins may be associated at that site (Fig. 1G).

\section{ADAM22 and ADAM23 modulate LGI1 targeting at the AIS of hippocampal neurons}

Strikingly, co-transfection with ADAM22 or ADAM23 at DIV7 strongly increased the somato-dendritic and axonal compartment cell surface expression of LGI1-GFP 1 day later (Fig. 2A,B, green; $\mathrm{C}$, red). Indeed LGI1-GFP expressed alone displayed a fluorescence intensity AIS:axon ratio of $3.26 \pm 0.23(n=16$; mean \pm s.e.m.). This ratio was significantly reduced to $1.38 \pm 0.11(n=24)$ and $1.15 \pm 0.10$ $(n=19)$ when co-transfected with ADAM22 and ADAM23, respectively (Fig. 2F). As a control experiment, we examined the polarized distribution of NrCAM-GFP since NrCAM does not belong to the Kv1 complex and is strongly recruited to the AIS by its ankyrinG-binding motif (Davis et al., 1996). NrCAM-GFP displayed an AIS:axon ratio of $4.05 \pm 0.6(n=20)$ and $5.51 \pm 0.9$ $(n=15)$ in the absence or presence of ADAM22, respectively (Fig. 2F). Therefore, the co-expression of ADAM22 did not modify the AIS distribution of NrCAM as illustrated in Fig. 2D,E.

Next, the ADAM23 sequence was fused to mCherry at its C-terminal to facilitate its fluorescent detection (red) and it was surface labeled using a rabbit anti-ADAM23 antibody (green) (Fig. S1B). ADAM23-mCherry exhibited a non-polarized surface expression when transfected alone (Fig. S1B) or when cotransfected with LGI1-GFP (Fig. S1C). It displayed an AIS:axon ratio of $1.58 \pm 0.13(n=11)$ (Fig. S1D). Moreover, LGI1-GFP colocalized with ADAM23-mCherry in clusters both along

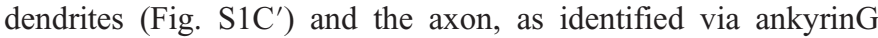
labeling (Fig. S1C"), in contrast with its AIS-restricted distribution when transfected alone (Fig. S1A).

We asked whether the recruitment of LGI1-GFP to the AIS may be correlated with the concentration of Kv1 channels at the AIS. In contrast to the early appearance of ankyrinG, NrCAM and voltagegated $\mathrm{Na}^{+}$channels, the Kv1.1 and Kv1.2 subunits (also known as $\mathrm{KCNA} 1$ and KCNA2, respectively) are tethered at the AIS of cultured hippocampal neurons only after 10 days in vitro (SánchezPonce et al., 2012; Vacher and Trimmer, 2012; Vacher et al., 2011). We analyzed neurons transfected with LGI1-GFP or co-transfected with LGI1-GFP and ADAM23-mCherry at DIV13, and the expression of endogenous Kv1.2 was measured at the AIS and along the axon 1 day later (Fig. 2G-J). When LGI1-GFP was transfected alone, it was enriched at the AIS and colocalized with Kv1.2 (Fig. 2G). The ratio of the mean fluorescence intensity at the AIS versus the axon was $2.82 \pm 0.3$ for LGI1-GFP and $2.47 \pm 0.24$ for Kv1.2. Individual values $(n=13)$ were plotted, showing that the AIS: axon ratio for LGI1-GFP could be correlated with that for the Kv1.2 channels (Fig. 2I). In contrast, when expressed with ADAM23mCherry, LGI1-GFP was no longer enriched at the AIS of co-transfected neurons (Fig. 2H,J). Therefore, the interplay between ADAM proteins and LGI1 may contribute to modulate the composition of the Kv1 complex at the AIS.

Our data indicate that the ADAM proteins may increase LGI1GFP expression at the overall neuronal cell surface either by stabilizing the secreted glycoprotein at the cell membrane or by promoting its export along the secretory pathway. In addition, overexpression of ADAM proteins may reduce anchoring of LGIGFP at the AIS through competition with endogenous ADAM22 enriched at this axonal subdomain.

\section{ADAM22 and ADAM23 promote ER export of LGI1}

We analyzed whether the ADAM proteins could be involved in the intracellular trafficking of LGI1 in transfected HEK cells. We observed that LGI1-GFP was strongly retained in the ER (green) when transfected alone in HEK cells and was poorly detected at the cell surface in live immunolabeling experiments for GFP and Alexa Fluor 647-conjugated secondary antibody (blue) (Fig. 3A). In contrast, LGI1-GFP co-expressed with ADAM23-mCherry was faintly detected in the ER and as strongly labeled clusters at the cell surface (blue) (Fig. 3C). When co-transfected with ADAM22mCherry, LGI1-GFP was also detected at the cell surface (Fig. 3B). Thus, we hypothesized that the association with ADAM proteins may favor the ER exit of LGI1. 

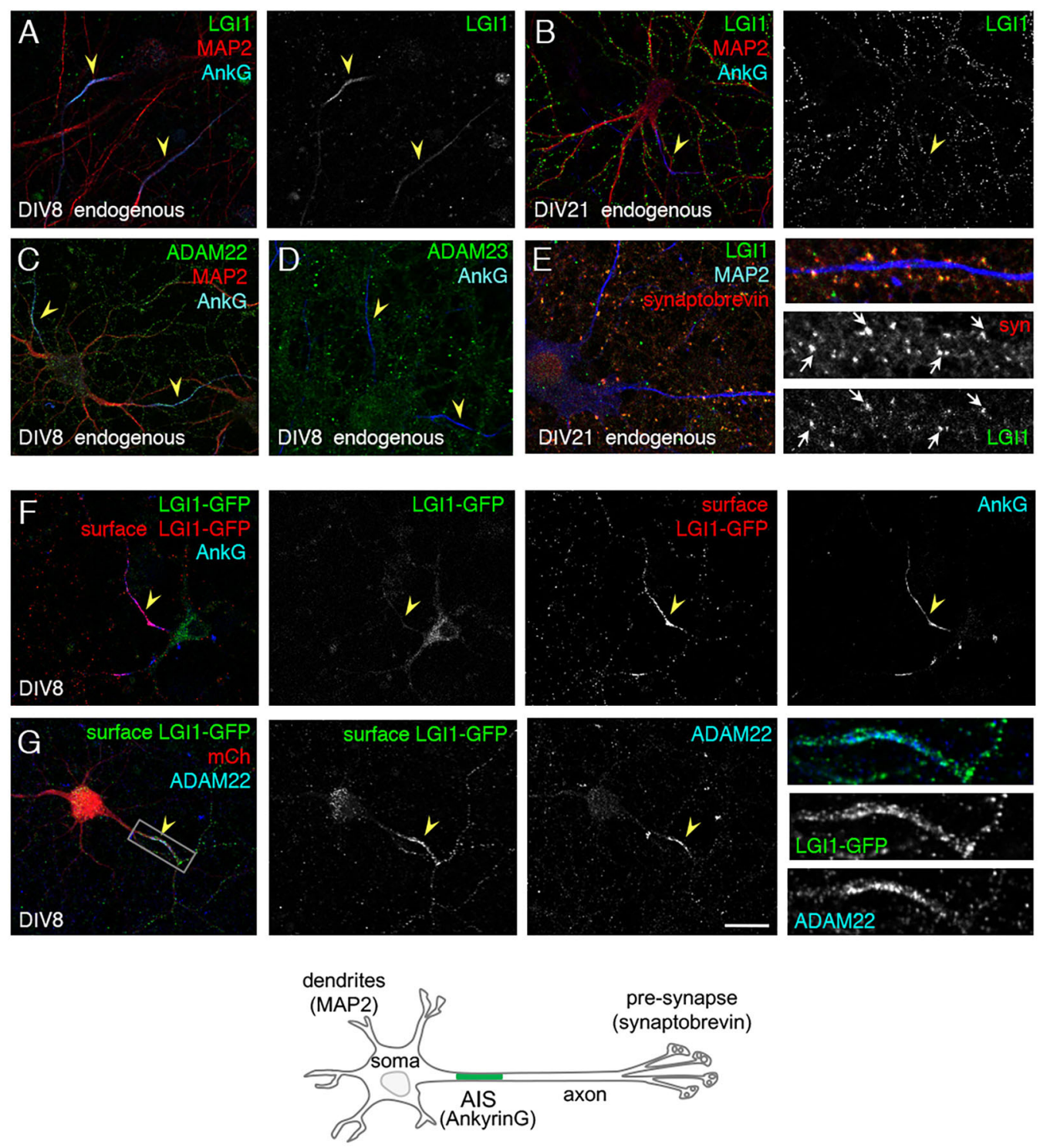

Fig. 1. LGI1 is enriched at the AIS in cultured hippocampal neurons. (A,B,E) Hippocampal neurons at DIV8 or DIV21 were surface labeled using anti-LGI1 mAb (green), and fixed and permeabilized before immunostaining for ankyrinG (A,B, blue) as a marker of the AIS (arrowheads) and MAP2, as a dendritic marker (A,B, red), or synaptobrevin (E, red), as a presynaptic marker, and MAP2 (E, blue). Note that endogenous LGI1 is only detected at the AIS at DIV8, whereas it is mainly distributed as puncta on the somato-dendritic compartment at DIV21. Arrows in E point to presynaptic terminals double-labeled for LGI1 and synaptobrevin. (C,D) DIV8 hippocampal neurons were fixed and permeabilized before immunostaining for ADAM22 (C) or ADAM23 (D), ankyrinG (blue) and MAP2 (red). Only ADAM22 is detected at the AIS (arrowheads), where it colocalized with ankyrinG. (F) Hippocampal neurons transfected at DIV7 with LGI1-GFP and immunostained at

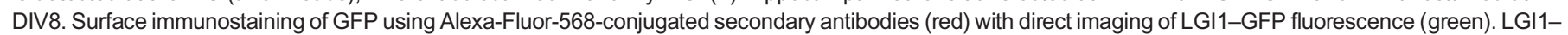
GFP surface labeling is restricted to the AIS, labeled with ankyrinG (blue). (G) Hippocampal neurons transfected at DIV7 with LGI1-GFP and mCherry. LGI1-GFP surface labeling (green) is colocalized at the AIS with endogenous ADAM22 (blue) immunostained after fixation and permeabilization. The AIS is indicated with yellow arrowheads. Scale bar: $20 \mu \mathrm{m}$. The diagram summarizes the markers used to identify the different neuronal compartments.

We examined the N-glycosylation processing of LGI1-GFP when expressed alone or in association with ADAM22 or ADAM23 in HEK cells. We analyzed the glycosylation pattern of LGI1-GFP by undertaking an experiments with endoglycanase $\mathrm{H}$ (Endo $\mathrm{H}$ ), which digests only immature ER-type high-mannose N-glycans. N-glycosidase F (PNGase F) was used to remove all the N-glycans. LGI1-GFP contained Endo H-sensitive carbohydrates when transfected alone (Fig. 3G). In contrast, when co-transfected with ADAM22 or ADAM23, two bands of LGI1-GFP were detected after treatment with Endo H, the higher band being Endo H-resistant and the lower band Endo H-sensitive form (Fig. 3G). This result indicates that ADAM22 and ADAM23 favor ER exit of LGI1, likely by acting as chaperone-like proteins through the ER quality-control system in HEK cells. In accordance with the results of Yokoi et al. (2015), we observed that Endo $\mathrm{H}$ digestion produced a mobility shift of LGI1-GFP while PNGase F treatment induced a further mobility shift of the same pool, as an indication that LGI1 can be partially processed in the absence of ADAM proteins.

\section{The secreted mutants LGI1S473L and LGI1 ${ }^{\mathrm{R474Q}}$ are not targeted to the AIS and display reduced interaction with ADAM22}

Human epilepsy-related missense mutations of LGI1 have been reported and classified as secretion-defective or secretioncompetent mutations. Among this last category, LGI1 ${ }^{\mathrm{S} 473 \mathrm{~L}}$, which is mutated in the EPTP domain, displays a reduced ability to bind to 

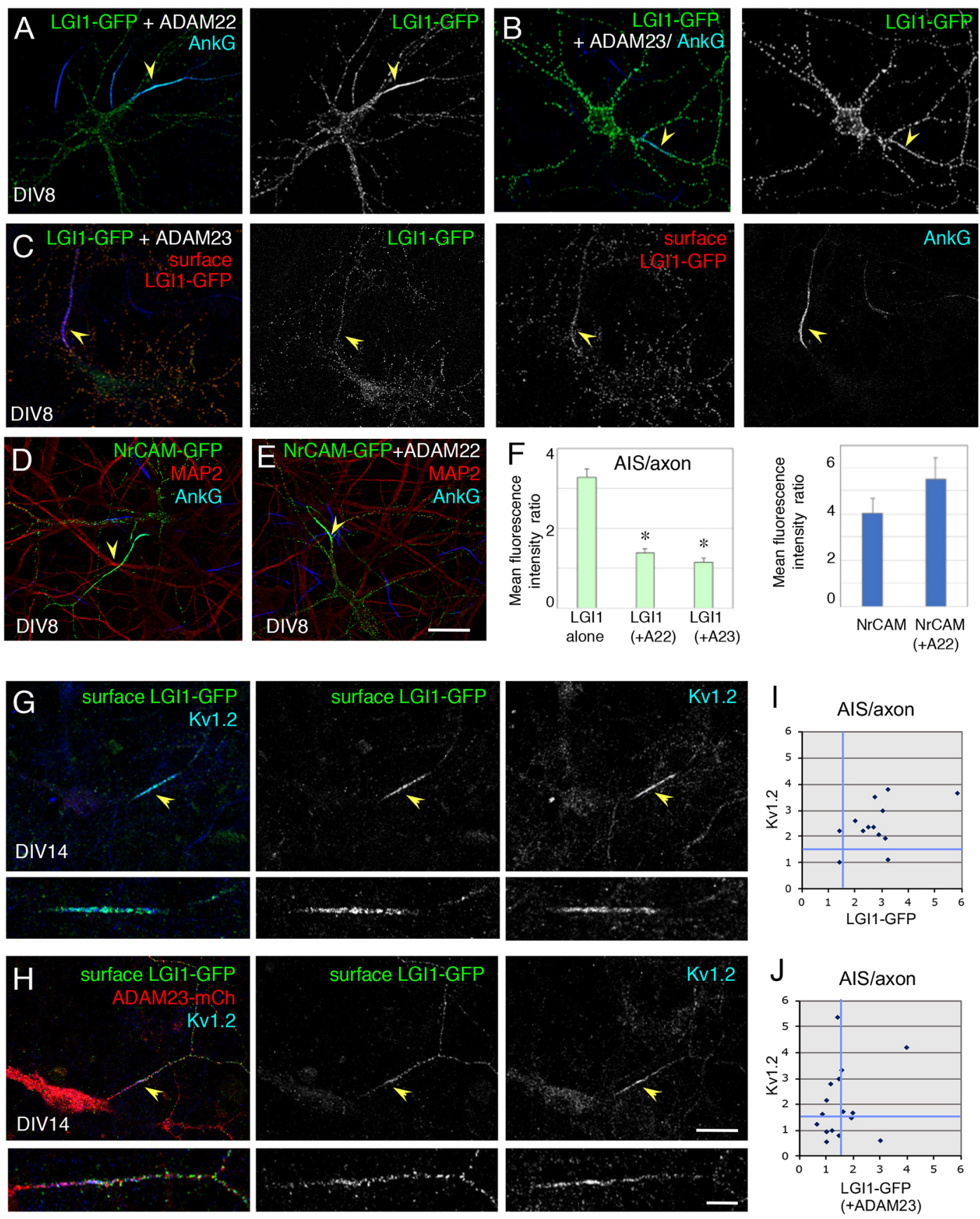

Fig. 2. Co-expression of ADAM22 or ADAM23 modulates the targeting of LGI1 at the AIS in hippocampal neurons. (A-F) Hippocampal neurons were transfected at DIV7 with LGI1-GFP and ADAM22 (A) or ADAM23 (B,C), NrCAM (D), or NrCAM and ADAM22 (E). Neurons were surface labeled 1 day later using anti-GFP antibodies (A,B,D,E, green; C, red), and fixed and permeabilized before immunostaining for ankyrinG (blue, arrowheads). Co-transfection with ADAM22 (A) or ADAM23 (B,C) strongly enhances LGI1-GFP expression at the somato-dendritic and axonal surface. Co-transfection with ADAM22 has no effect on NrCAM distribution at the AIS (D,E). (F) Ratios of fluorescence intensity between the AIS and axon in neurons transfected at DIV7 with LGI1-GFP alone, NrCAMGFP alone, or co-transfected with ADAM22 (A22) or ADAM23 (A23). Results are mean \pm s.e.m., $n=15-24$. * indicates significant differences by comparison with LGI1-GFP transfected alone for co-transfection with ADAM22 or ADAM23 $[F(2,56)=55.68$, ANOVA and $P<0.01$, Fisher test]. The co-expression of ADAM22 did not modify the AIS distribution of NrCAM-GFP (Mann-Whitney test, $P=0.13$ ). (G-J) The AIS enrichment in LGI1-GFP correlated with AIS expression of endogenous Kv1.2. DIV13 hippocampal neurons were transfected with LGI1-GFP (G) or with LGI1-GFP and ADAM23-mCherry (H). Neurons were surface labeled 1 day later for GFP (green), and fixed, permeabilized and immunostained for Kv1.2 (blue). Note that LGI1-GFP is strongly colocalized with Kv1.2 at the AIS in a neuron transfected with LGI1-GFP alone, but not in a neuron co-transfected with LGI1-GFP and ADAM23-mCherry. The AIS is indicated with an arrowhead. (I,J) The AIS:axon ratios of fluorescence intensity for LGI1-GFP and Kv1.2 were plotted for individual neurons when transfected with LGI1-GFP or co-transfected with LGI1-GFP and ADAM23-mCherry. Scale bar: $20 \mu \mathrm{m}$ (main images), $5 \mu \mathrm{m}$ (magnifications).

ADAM22 but not ADAM23 (Yokoi et al., 2015). Therefore, we asked whether LGI1 ${ }^{\mathrm{S} 473 \mathrm{~L}}$ could be properly targeted to the AIS of hippocampal neurons when expressed alone or in combination with ADAM22 or ADAM23. First, we analyzed the processing of
LGI1 ${ }^{\text {S473L }}$ in HEK cells and observed that both ADAM22 and ADAM23 induced its cell surface expression (Fig. 3D-F). Western blotting experiments indicated that $\mathrm{LGI}{ }^{\mathrm{S} 473 \mathrm{~L}}$ displayed Endo $\mathrm{H}$-sensitive $\mathrm{N}$-glycans when expressed alone in HEK cells, showing 

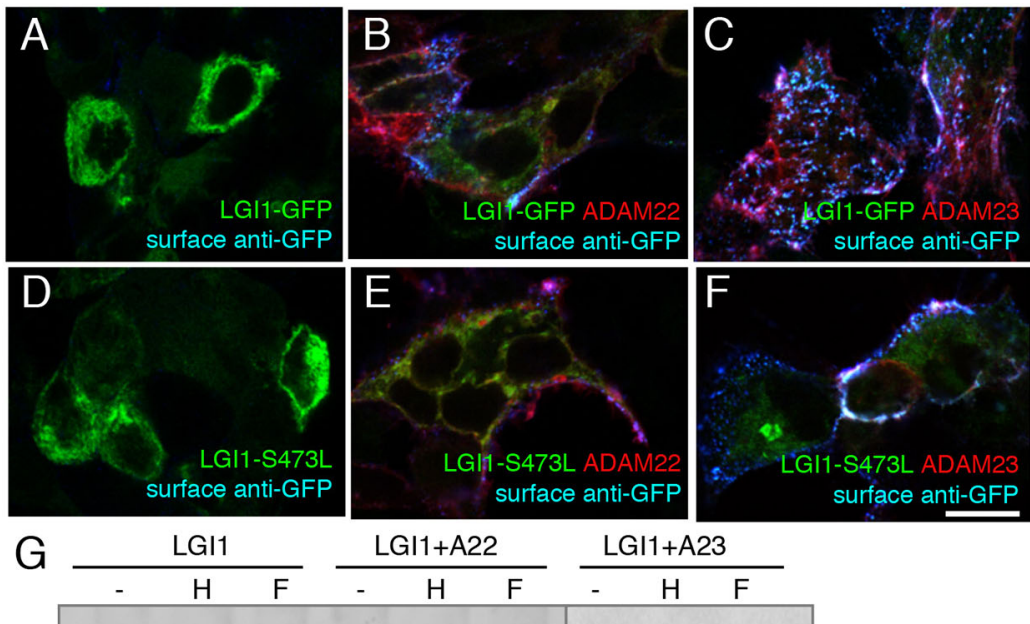

$\frac{\mathrm{LG} 11+\mathrm{A} 22}{\mathrm{H} F} \frac{\mathrm{LG} 1 \text { 1+A23 }}{-}$
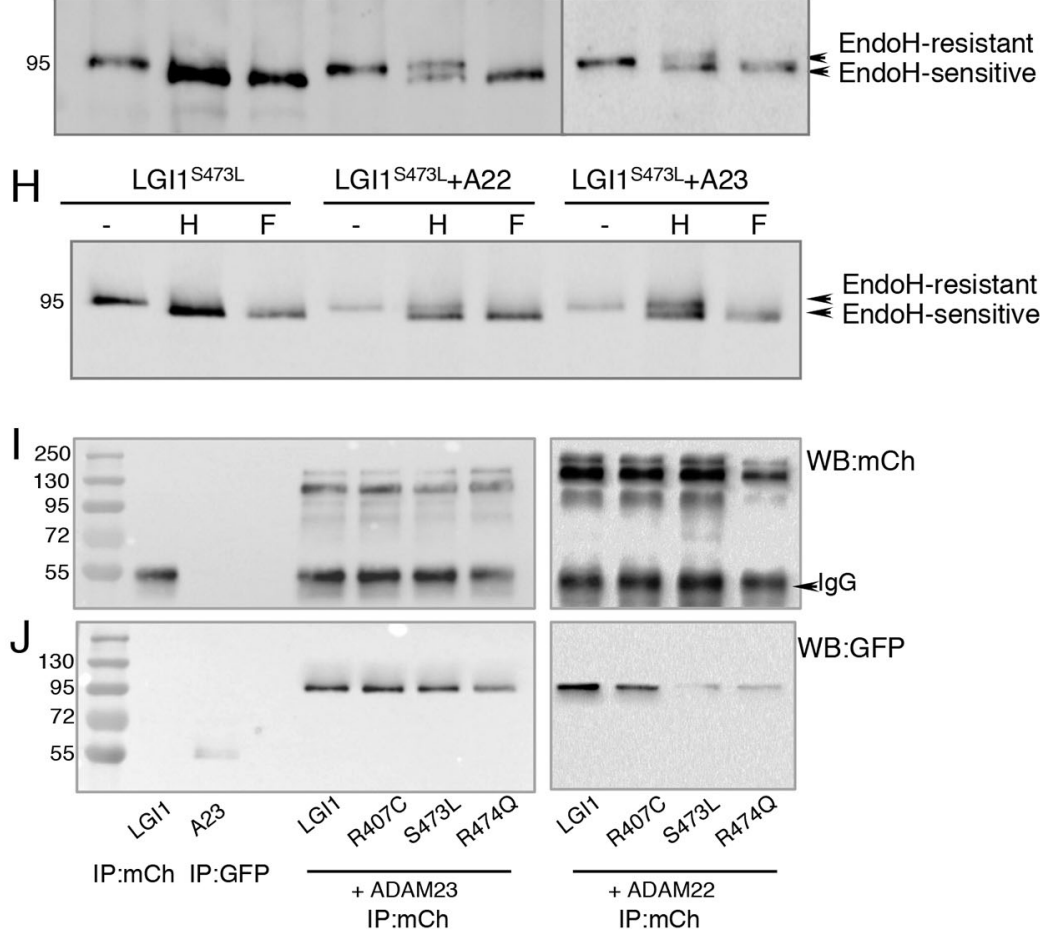

K

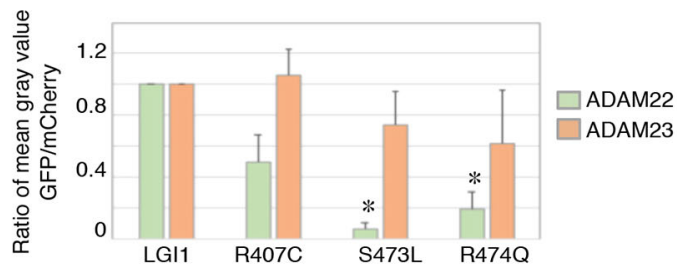

the same mobility shift as the wild-type LGI1-GFP after Endo H or PNGase $\mathrm{F}$ treatment (Fig. 3H). LGI1 ${ }^{\mathrm{S} 473 \mathrm{~L}}$ co-expressed with ADAM22 or ADAM23 displayed both Endo H-resistant and Endo H-sensitive glycoforms (Fig. 3H). Thus, LGI1 ${ }^{\text {S473L }}$ still had the capacity of interacting in cis with ADAM proteins, which enhanced its ER exit and processing with complex N-glycans in HEK cells.

Strikingly, when transfected in hippocampal neurons at DIV7, LGI1 ${ }^{\mathrm{S} 473 \mathrm{~L}}$ was not enriched at the AIS (Fig. 4C) in contrast to wild-type LGI1-GFP (Fig. 4A). The mean AIS:axon ratios were $3.62 \pm 0.39(n=20)$ for LGI1-GFP and $1.18 \pm 0.09(n=17)$ for LGI1 $^{\text {S473L }}$. Co-transfection with ADAM22- or ADAM23mCherry strongly enhanced the surface labeling of LGI1 ${ }^{\mathrm{S} 473 \mathrm{~L}}$, which became unpolarized at the neuronal cell surface (Fig. 4G,H).
Fig. 3. ADAM proteins promote ER exit and $\mathrm{N}$-glycan maturation of LGI1 and $\mathrm{LGI} 1^{\mathrm{S} 473 \mathrm{~L}}$ and differentially associate with LGI1 mutants. HEK cells (A-F) were transfected with LGI1-GFP alone (A), or co-transfected with ADAM22- (B) or ADAM23-mCherry (C), transfected with

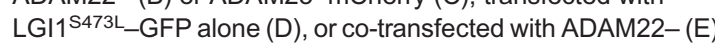
or ADAM23-mCherry (F). The fluorescence for GFP was directly imaged (green) to visualize the intracellular pool of LGI1 whereas the surface pool was labeled using anti-GFP antibody (blue). Note that ADAM22 and ADAM23 promote ER exit and

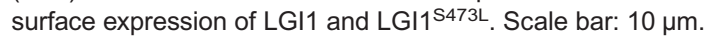
$(G, H)$ HEK cells were transfected with LGI1-GFP $(G)$ or

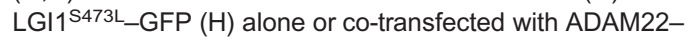
mCherry or ADAM23-mCherry. After cell lysis, wild-type or mutated LGI1 was immunoprecipitated using anti-GFP mAb and incubated at $37^{\circ} \mathrm{C}$ for $3 \mathrm{~h}$ without (-) or with Endo $\mathrm{H}$ (denoted with an $\mathrm{H}$ ) or PNGase $\mathrm{F}$ (denoted with an $\mathrm{F}$ ). Western blotting with anti-GFP mAb shows that LGI1-GFP migrates as a doublet when co-transfected with ADAM22 or ADAM23 and incubated with Endo $\mathrm{H}$. Note that the lower band corresponding to the Endo $\mathrm{H}$-sensitive glycoform migrates in the same position as LGI1-GFP treated with PNGase F. The higher band, which is Endo $\mathrm{H}$-resistant, migrated in the same position as untreated LGI1-GFP. The LGI1 ${ }^{\mathrm{S} 473 \mathrm{~L}}$ mutant also displayed Endo $\mathrm{H}$-sensitive and resistant glycoforms. (I,J) HEK cells were transfected with LGI1-GFP (lane 1) or ADAM23-mCherry (lane 2) alone, or co-transfected with GFP-tagged LGI1, LGI1 ${ }^{\mathrm{R} 407 \mathrm{C}}, \mathrm{LGI} 1^{\mathrm{S} 473 \mathrm{~L}}$ or $\mathrm{LGI} 1^{\mathrm{R} 474 \mathrm{Q}}$ and ADAM22-mCherry or ADAM23-mCherry. After cell lysis, ADAM proteins were immunoprecipitated using rabbit anti-mCherry. Western blotting was performed using rabbit anti-mCherry (I) and anti-GFP antibodies $(\mathrm{J})$ to reveal co-immunoprecipitated proteins. Experiments were performed in triplicate. (K) Quantitative analysis of co-immunoprecipitated and immunoprecipitated proteins was performed using the Image J software and expressed as a percentage of the wild-type LGI1 value. Results are mean \pm s.e.m., $n=3 .{ }^{*}$ indicates significant differences of the ratio of co-precipitated LGI1 mutant relative to the amount of immunoprecipitated ADAM22 by comparison with wild-type LGI1 using ANOVA $(P<0.021)$ and $t$-test $(P=0.0011$ and $P=0.0135$ for $L G \mid 1^{\mathrm{S} 473 \mathrm{~L}}$ or $\mathrm{LGI} 1^{\mathrm{R} 474 \mathrm{Q}}$, respectively).
These data indicate that the binding activity of LGI1 ${ }^{\mathrm{S} 473 \mathrm{~L}}$ for ADAM22 and ADAM23 allows its transport to the cell surface in hippocampal neurons. Next, we tested the distribution of two other secreted mutants of LGI1, LGI1 ${ }^{\mathrm{R} 407 \mathrm{C}}$ and $\mathrm{LGI}^{\mathrm{R} 474 \mathrm{Q}}$ when transfected in DIV7 hippocampal neurons. As expected, the R474Q mutation, which is adjacent to the S473L mutation also prevented the AIS enrichment of LGI1 (Fig. 4D,J). In contrast, LGI ${ }^{\mathrm{R} 407 \mathrm{C}}$ was enriched at the AIS of hippocampal neurons as observed for the wild-type LGI1 (Fig. 4B,J). The mean AIS:axon ratios were $1.55 \pm 0.13(n=17)$ for $\mathrm{LGI} 1^{\mathrm{R} 474 \mathrm{Q}}$ and $2.72 \pm 0.19(n=19)$ for LGI1 ${ }^{\mathrm{R} 407 \mathrm{C}}$. Co-transfection with ADAM22- or ADAM23mCherry strongly enhanced the neuronal cell surface expression of LGI1 ${ }^{\mathrm{R} 474 \mathrm{Q}}$ (data not shown) and $\mathrm{LGI}^{\mathrm{R} 407 \mathrm{C}}$ (Fig. 4E,F). In 

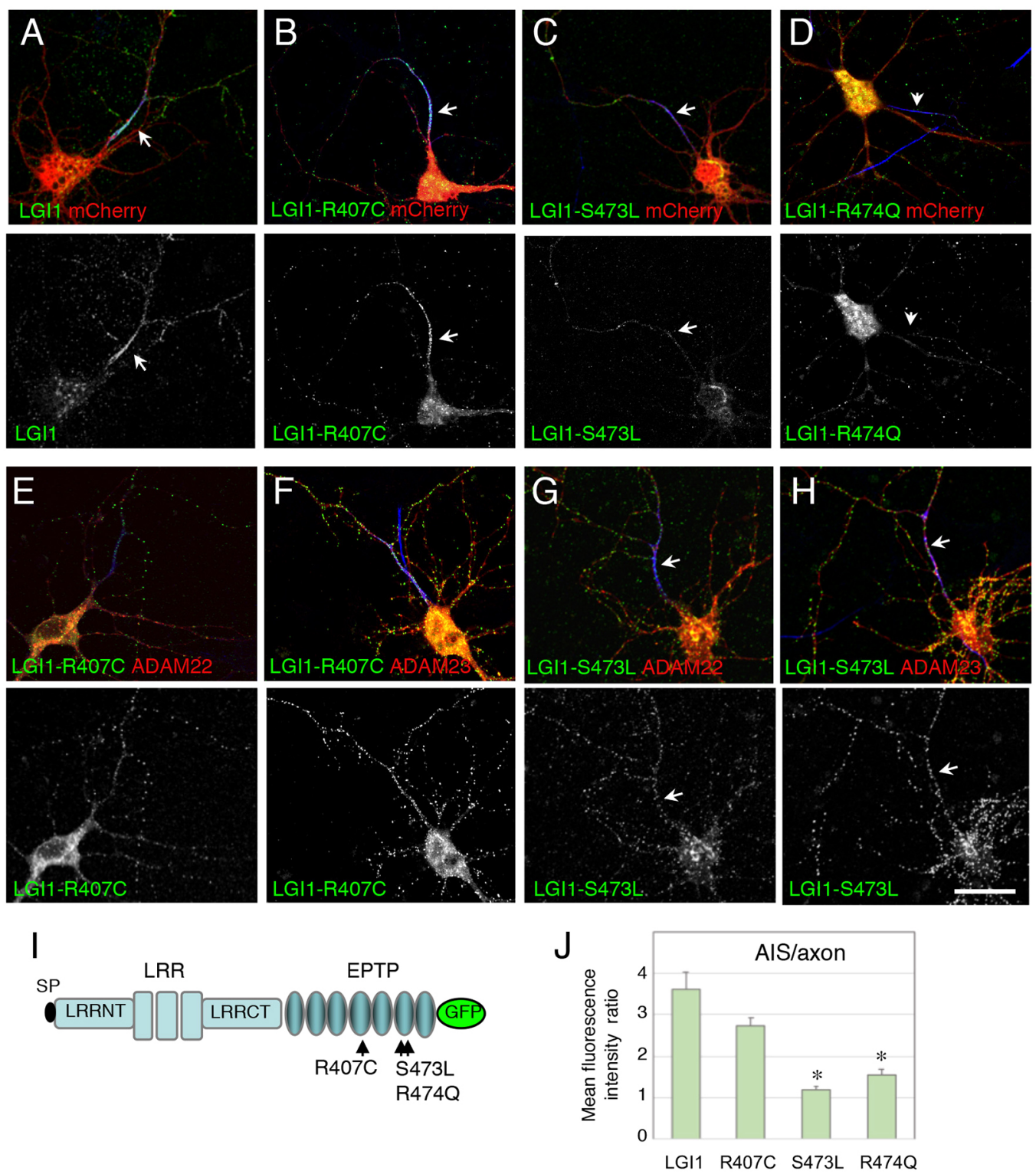

Fig. 4. The variants of LGI1 associated with epilepsy are differentially recruited at the AIS of hippocampal neurons. DIV7 hippocampal neurons were transfected with GFP-tagged LGI1 (A), LGI1 ${ }^{\mathrm{R} 407 \mathrm{C}}(\mathrm{B}), \mathrm{LGI} 1^{\mathrm{S} 473 \mathrm{~L}}(\mathrm{C})$ and $\mathrm{LGI} 1^{\mathrm{R} 474 \mathrm{Q}}(\mathrm{D})$. Note that both $\mathrm{LGI} 1^{\mathrm{S} 473 \mathrm{~L}}$ and $\mathrm{LGI} 1^{\mathrm{R} 474 \mathrm{Q}}$ are not enriched at the $A \mid \mathrm{S}$ in contrast to wild-type LGI1 and LGI1 ${ }^{\text {R407C }}$. GFP-tagged LGI1 ${ }^{\mathrm{R} 407 \mathrm{C}}$ or LGI1 ${ }^{\mathrm{S} 473 \mathrm{~L}}$ were co-transfected with ADAM22- (E,G) or ADAM23-mCherry $(\mathrm{F}, \mathrm{H})$. When co-transfected with ADAM22- or ADAM23-mCherry, the neuronal surface expression of all the variants is strongly increased. (I) Schematic representation of LGI1 with the point mutations localized in the EPTP domains. LRR, leucine-rich repeats; LRRNT, N-terminal LRR; LRRCT, C-terminal LRR. (J) Ratios of fluorescence intensity between AIS and axon in neurons transfected at DIV8 with GFP-tagged LGI1, LGI1 ${ }^{\mathrm{R} 407 \mathrm{C}}$, LGI ${ }^{\mathrm{S} 473 \mathrm{~L}}$ or LGI1 ${ }^{\mathrm{R} 474 \mathrm{Q}}$. Results are mean \pm s.e.m., $n=17-20$. * indicates significant differences by comparison with wild-type LGI1-GFP $(P<0.0001$, Mann-Whitney test). Scale bar: $20 \mu \mathrm{m}$.

conclusion, two missense mutations located in the EPTP6 domain, the S473L and R474Q mutations, both impair the recruitment of LGI1 to the AIS as a possible pathogenic mechanism. In contrast, the mutation R407C in the EPTP4 domain had no effect on AIS targeting (Fig. 4I).

The association of the three LGI1 mutants with co-transfected ADAM22 and ADAM23 is sufficient to promote their expression at the neuronal cell surface. However, an altered interaction with ADAM22, which is anchored at the AIS of hippocampal neurons, may prevent their proper targeting at that site. Using co-immunoprecipitation experiments from transfected HEK cells, we analyzed the association of the secreted LGI1 mutants LGI1 ${ }^{\mathrm{R} 407 \mathrm{C}}$, LGI1 ${ }^{\mathrm{S} 473 \mathrm{~L}}$ and $\mathrm{LGI1}^{\mathrm{R} 474 \mathrm{Q}}$ with $\mathrm{ADAM} 22_{-}$or ADAM23-mCherry (Fig. 3I,J). Single transfections with
LGI1-GFP or ADAM23-mCherry were used as negative controls. All the secreted mutants were co-immunoprecipitated with ADAM23-mCherry as was the case for the wild-type LGI1GFP (Fig. 3J, left panel, K). However, the S473L and R474Q mutations significantly reduced the co-immunoprecipitation of LGI1 with ADAM22-mCherry (Fig. 3J, right panel, K). These results suggest that these missense mutations may impair the anchorage of LGI1 to ADAM22 at the AIS of hippocampal neurons.

To investigate whether ADAM22 may be implicated in trapping LGI1 at the AIS, we tested the effect of ADAM22 depletion using siRNA transfection. Hippocampal neurons were transfected at DIV7 with LGI1-GFP and control, ADAM23-, or ADAM22specific siRNAs and analyzed 4 days later via immunofluorescence staining (Fig. 5). We observed that the amount of ADAM22 was 

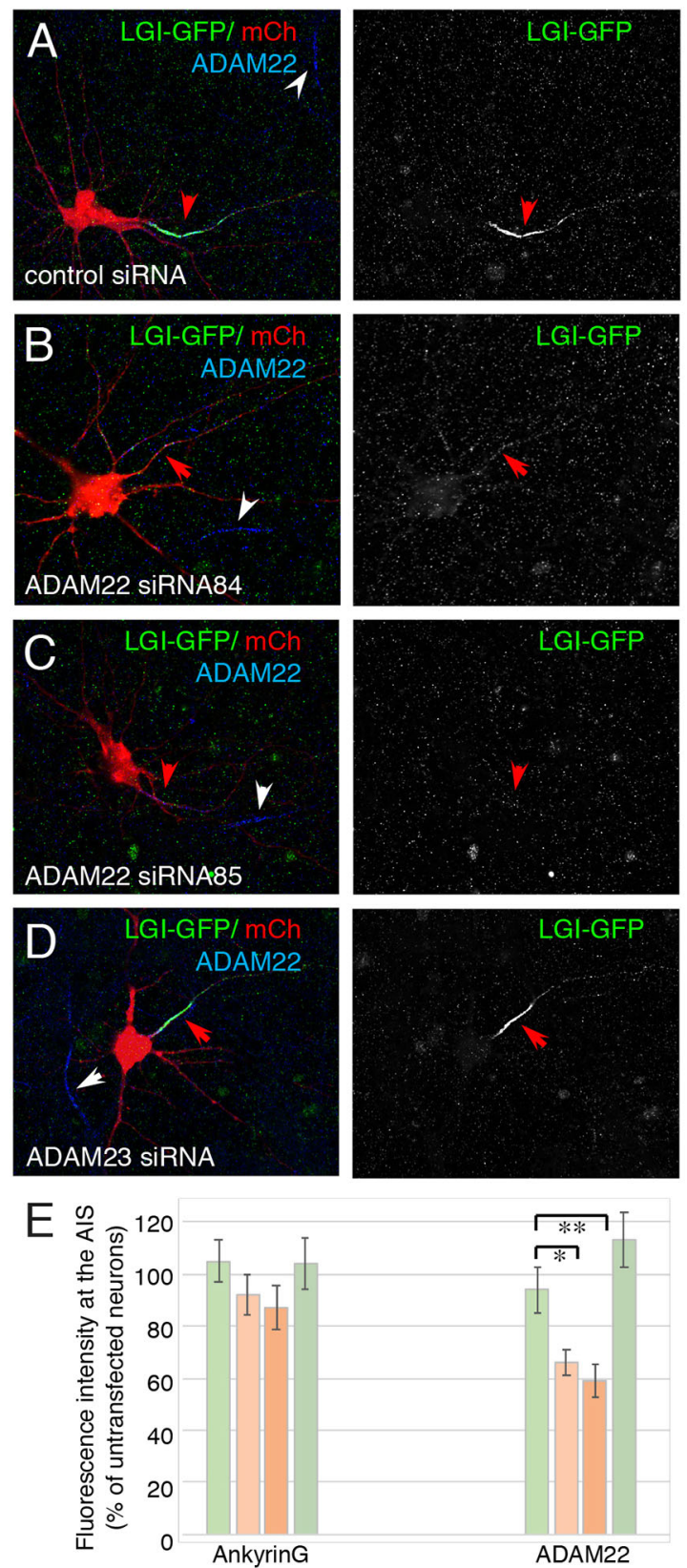
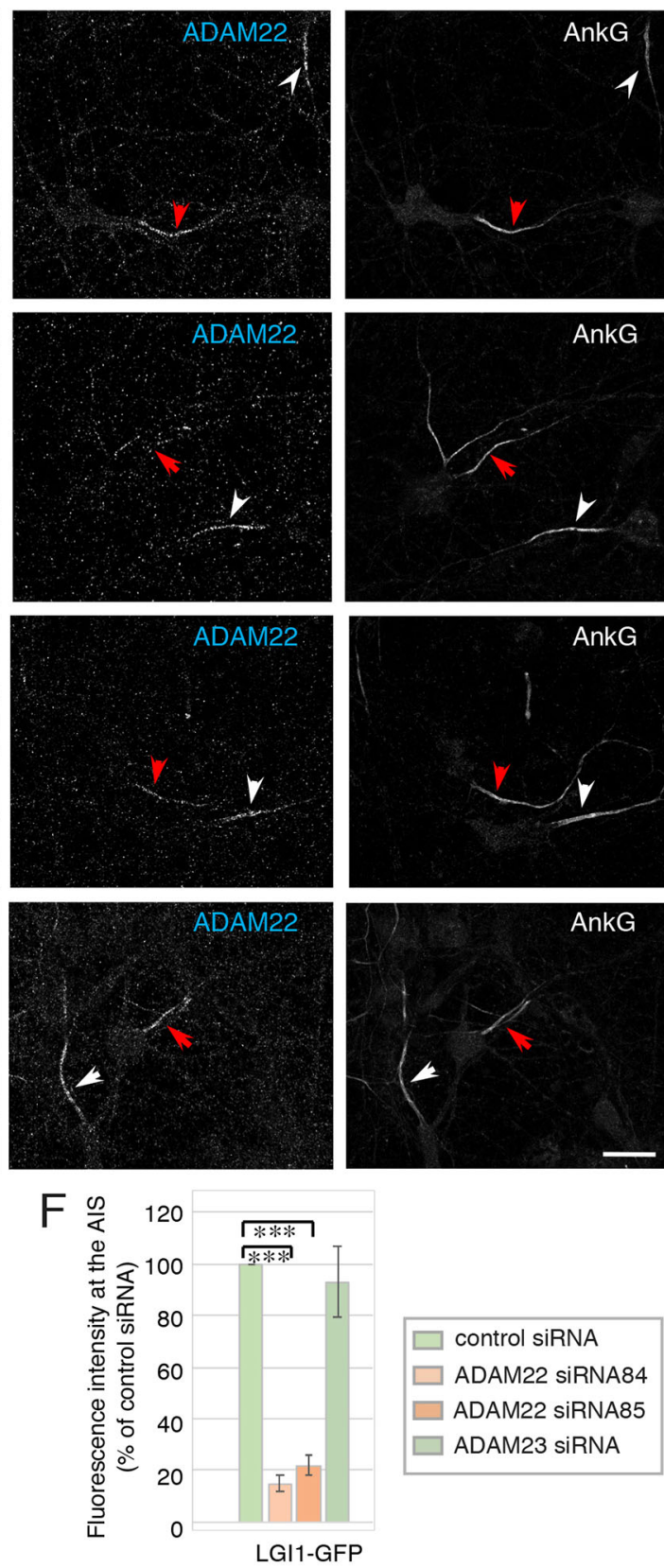

Fig. 5. siRNA-mediated silencing of ADAM22 prevents the expression of LGI1-GFP at the AIS of hippocampal neurons. Hippocampal neurons were transfected at DIV7 with LGI1-GFP, mCherry and control- (A), ADAM22 (B,C) or ADAM23 siRNAs (D). Cells were surface labeled at DIV11 using anti-GFP antibody (Alexa Fluor 488, green), and fixed and permeabilized before immunostaining for ADAM22 (Alexa Fluor 647, blue) and ankyrinG (Alexa Fluor 405, white). LGI1-GFP is highly recruited at the AIS of neurons transfected with control (A) or ADAM23 siRNA (D), whereas it is hardly detectable in the presence of ADAM22 siRNAs (B,C). Note that ADAM22 is still detected at the AIS in cells transfected with ADAM22 siRNAs (B,C), but with a lower fluorescence

intensity than in the untransfected neurons. Red and white arrowheads indicate the AIS of transfected and untransfected neurons, respectively. Scale bar: $20 \mu \mathrm{m}$. (E) Mean \pm s.e.m. fluorescence intensity for ankyrin G and ADAM22 at the AIS of transfected neurons as a percentage of the value for 2-3 untransfected neurons in the same areas (11-15 areas were imaged using identical confocal parameters under each condition). ${ }^{*} P=0.0251,{ }^{* *} P=0.0086$ by comparison with control siRNA (Mann-Whitney test). (F) Mean \pm s.e.m. $(n=11-15)$ fluorescence intensity for LGI1-GFP at the AIS of transfected neurons as a percentage of the value for control siRNA. ${ }^{* * *} P<0.0001$ by comparison with control siRNA $[F(3,45)=16.03$, ANOVA].

significantly decreased, by $30-37 \%$, at the AIS of neurons transfected with two different ADAM22-specific siRNAs (s186484 and s186485) by comparison with non-transfected neurons in the same areas (Fig. 5B,C,E). Strikingly, LGI1-GFP immunofluorescence intensity at the AIS was almost completely abolished (78-85\% reduction) in neurons transfected with ADAM22 siRNAs by comparison with neurons transfected with control or ADAM23-specific siRNAs (Fig. 5F). Taken together, these results indicate that ADAM22 is critical for the enrichment of LGI1-GFP at the AIS.

\section{Axonal transport of ADAM22, ADAM23 and LGI1}

We next investigated whether LGI1 could be associated with ADAM proteins in axonal transport vesicles. First, we performed time-lapse imaging of neurons transfected at DIV7 with ADAM22mCherry or ADAM23-mCherry to get insights into their axonal 
targeting mechanisms. The axon was clearly identified on the basis of its length (and was strongly enriched in transport vesicles by comparison with dendrites). In addition, live immunolabeling of neurofascin isoform 186 (NF186) was used to precisely localize the AIS after time-lapse recording (Fig. 6A,B, blue). In neurons that were transfected with ADAM22-mCherry, we observed that labeled vesicles were axonally transported in the anterograde and retrograde directions with a maximal velocity $\left(V_{\mathrm{m}}\right)$ of $0.69 \pm 0.1$ and $0.51 \pm 0.06 \mu \mathrm{m} \mathrm{s}^{-1}$, respectively (Table S1; Movie 1). In ADAM23mCherry-transfected neurons, labeled vesicles were transported in the anterograde and retrograde directions with a $V_{\mathrm{m}}$ of $0.99 \pm 0.11$ and $0.53 \pm 0.06 \mu \mathrm{m} \mathrm{s}^{-1}$, respectively (Table S1; Movie 2). However, ADAM22 vesicles moved bi-directionally in most neurons (Table S1, $n=10$ neurons) whereas, by contrast, ADAM23 vesicles were mostly transported in the anterograde direction $(65.2 \pm 9.5 \%$ of displacements, $n=9$ neurons; mean \pm s.e.m.) as

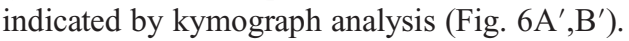

Next, we performed two-color time-lapse imaging of neurons co-transfected with ADAM23-mCherry and LGI1-GFP to visualize their axonal transport (Fig. 6C-E). We found that most of the axonal transport vesicles were colabeled for LGI1 and ADAM23. Kymograph analysis of transport events indicated that
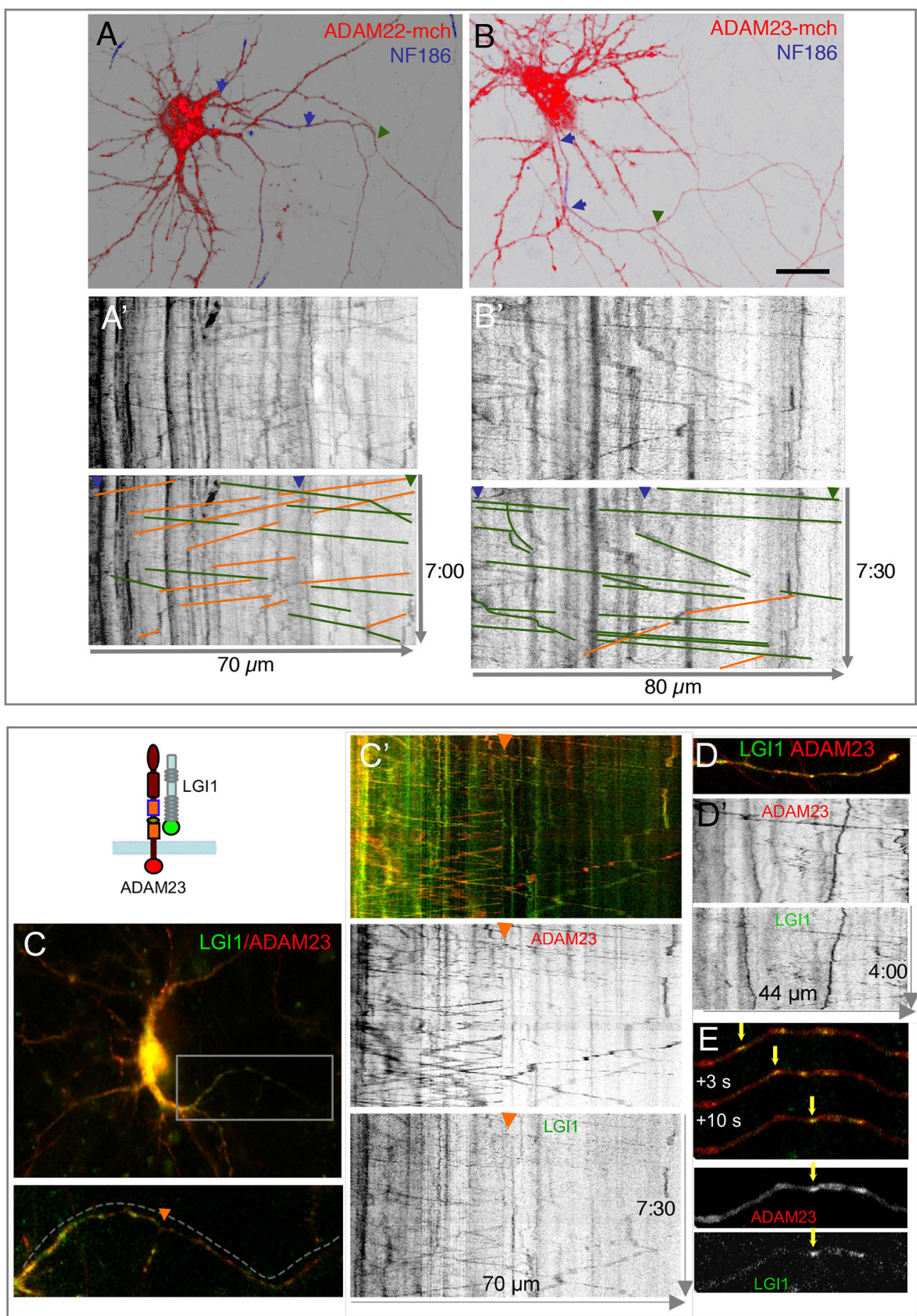

Fig. 6. LGI1 and ADAM23 are colocalized in transport vesicles. $(A, B)$ Hippocampal neurons were transfected at DIV7 with ADAM22mCherry (A) or ADAM23-mCherry (B). Time-lapse images of axonal transport vesicles were acquired 1 day later at 1 frame per $1.5 \mathrm{~s}$. Live immunostaining with Alexa Fluor 647-coupled antiNeurofascin186 was performed to determine AIS location (blue) limited with blue arrowheads. $\left(A^{\prime}, B^{\prime}\right)$

Corresponding kymographs with axonal length in $x$-axis and time in $y$-axis. Anterograde and retrograde events are highlighted with green and orange traces, respectively. The maximal velocity was measured for each transport sequence. Note that transport events were mostly in the retrograde direction for ADAM22 whereas they were mostly in the anterograde direction for ADAM23. See the corresponding Movies 1 and 2. (C-E) Hippocampal neurons were co-transfected with LGI1GFP and ADAM23-mCherry at DIV8. Live-cell recording of proximal (C) and distal $(D, E)$ axons. The orange arrowhead labels a proximal axon bifurcation in $C, C^{\prime}$. $\left(C^{\prime}, D^{\prime}\right)$ The corresponding kymographs show overlapping trajectories of vesicles labeled for LGI1-GFP and ADAM23mCherry. Comparison of traces for transport events along the axon indicated that a number of vesicles were colabeled with LGI1-GFP and ADAM23-mCherry moving both in anterograde and retrograde directions. (E) Time-lapse sequence showing a moving vesicle that contains both LGI1-GFP and ADAM23-mCherry, indicated with arrows. Movies 3 and 4 show time-lapse recordings of proximal and distal axons, respectively. Scale bars: $20 \mu \mathrm{m}$. 
double-labeled vesicles moved bi-directionally, as illustrated in proximal (Fig. 6C, $\mathrm{C}^{\prime}$ ) or distal (Fig. 6D,E) axonal regions (Movies 3 and 4). The $V_{\mathrm{m}}$ for the anterograde and retrograde transports was $0.87 \pm 0.09$ and $0.86 \pm 0.08 \mu \mathrm{m} \mathrm{s}^{-1}$, respectively. We observed mostly anterograde events ( $74 \pm 4.7 \%$ of displacements, $n=4$ neurons), as occurring in neurons transfected with ADAM23 alone. In neurons co-transfected with ADAM22-mCherry and LGI1-GFP, we also observed axonal vesicles colabeled for both CAMs that were mostly retrogradely transported (Fig. S2, $n=3$ neurons). Vesicles labeled for LGI1-GFP were not easily detected when transfected alone, further indicating that LGI1 may require co-expression with ADAM22 or ADAM23 for its proper trafficking and axonal transport.

\section{Biochemical interactions of ADAM22 and ADAM23 with the Kv1-associated CAMs TAG-1 and Caspr2}

Apart from LGI1 and ADAM proteins, another set of CAMs, including TAG-1 and Caspr2, has been reported to associate with Kv1 channels at discrete regions of the axon, and we investigated whether these different CAMs may interact in complex with the ADAM22 and ADAM23 proteins. Biochemical interactions between LGI1 and both ADAM22 and ADAM23 have been well documented; LGI1 interacts, via its EPTP repeats, with several members of the ADAM family, including ADAM22 and ADAM23, as reported using cell-binding assays and co-immunoprecipitation experiments (Fukata et al., 2006; Owuor et al., 2009; Sagane et al., 2008). Hence, we performed co-imunoprecipitation experiments from transfected HEK cells to investigate whether ADAM22 and ADAM23 could also interact with TAG-1 and Caspr2. As shown in Fig. 7A,C, using co-immunoprecipitation with anti-mCherry or anti-GFP antibodies, LGI1-GFP but not TAG-1-GFP could form a complex with ADAM23-mCherry. In addition, we showed that HA-tagged Caspr2 and Caspr2-GFP were co-immunoprecipitated with ADAM23-mCherry when using an anti-mCherry antibody (Fig. 7B,C). Moreover, Caspr2 with a deleted cytoplasmic tail (Caspr2 $\Delta$ cyt) was also efficiently co-immunoprecipitated with ADAM23, demonstrating that these membrane proteins interact via their ectodomains or transmembrane domains (Fig. 7B). Conversely, ADAM23 was not precipitated with HA-tagged Caspr2 when using anti-HA antibody (Fig. 7B). We note that mCherry is fused at the C-terminus of ADAM23 cytoplasmic tail whereas $\mathrm{HA}$ is placed at the $\mathrm{N}$-terminal region of Caspr2, so that it is possible that the anti-HA antibody could interfere with the

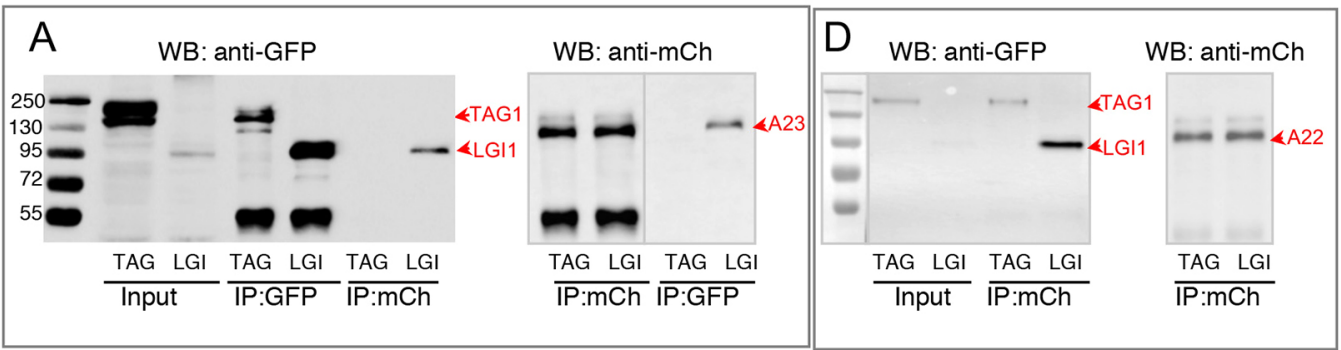

ADAM23-mCh + Caspr2-HA

ADAM22-mCh + Caspr2-HA
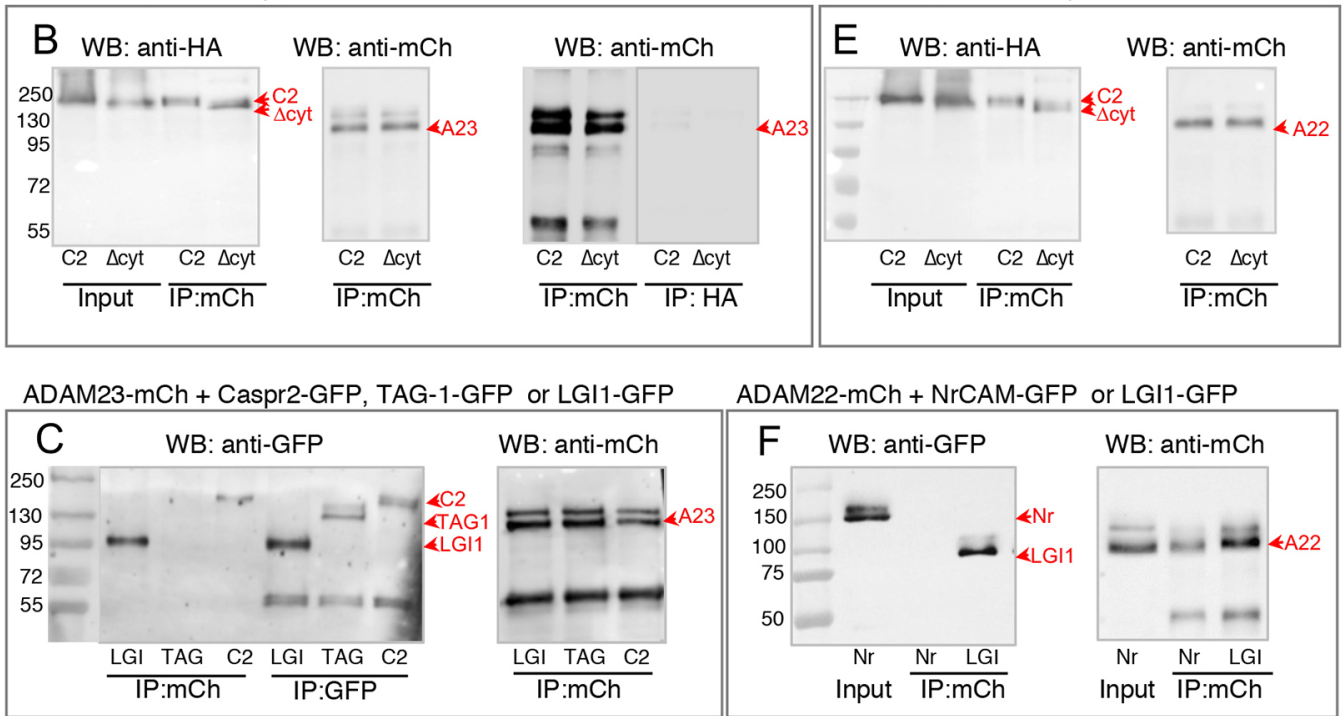

Fig. 7. Biochemical analysis of the interaction between ADAM proteins and LGI1, TAG-1 or Caspr2. Co-immunoprecipitation experiments from HEK cells transfected with ADAM23-mCherry (A-C) or ADAM22-mCherry (D-F), and TAG-1-GFP or LGI1-GFP (A,D), or Caspr2-HA constructs, either full-

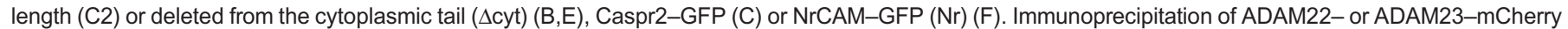
was performed using rabbit anti-mCherry antibody (IP mCh). Immunoprecipitation of TAG-1-GFP, Caspr2-GFP or LGI1--GFP was performed with mouse anti-GFP mAb (IP GFP). After western blotting, TAG-1, LGI1, Caspr2 and NrCAM constructs were detected using mouse anti-GFP mAb, ADAM proteins using rabbit anti-mCherry antibody, and Caspr2-HA constructs using rat anti-HA mAb in the lysates (input) and immunoprecipitates. LGI1-GFP was co-immunoprecipitated with ADAM22 (D) and ADAM23 (A). TAG-1 was co-immunoprecipitated with ADAM22 (D) but not with ADAM23 (A). Both full-length Caspr2 and Caspr2 $\Delta$ cyt were co-immunoprecipitated with ADAM22 (E) and ADAM23 (B,C). Reciprocally, ADAM23 was not detected after immunoprecipitation of Caspr2 using anti-HA mAb (B). NrCAM was not co-immunoprecipitated with ADAM22 (F). Experiments were performed in triplicate. 
binding between CAM ectodomains. In addition, we also showed that LGI1-GFP and TAG-1-GFP were co-immunoprecipitated with ADAM22-mCherry (Fig. 7D). Caspr2 and Caspr2 $\Delta$ cyt were similarly co-immunoprecipitated with ADAM22-mCherry when using an anti-mCherry antibody (Fig. 7E). By contrast, NrCAMGFP did not associate with ADAM22-mCherry (Fig. 7F) or ADAM23-mCherry (data not shown). Thus, when expressed in HEK cells, ADAM22 and ADAM23 display the capability to selectively associate with multiple CAMs related to the Kv1 complex.

\section{The axonal targeting of ADAM23 is modulated by its co-expression with TAG-1 and Caspr2}

We asked whether the ADAM proteins could interfere with the axonal targeting of the two components of the Kv1 complex, Caspr2 and TAG-1. We recently showed that both endogenous and transfected TAG-1 and Caspr2 are differentially distributed in cultured hippocampal neurons; TAG-1 is enriched at the AIS whereas Caspr2 is evenly localized along the axon (Pinatel et al., 2017). Neurons were co-transfected at DIV13 with TAG-1-GFP and ADAM23, and surface labeled using anti-GFP and antiADAM23 antibodies 1 day later. Strikingly, we observed that ADAM23 colocalized with TAG-1 at the neuronal surface and was enriched at the AIS (Fig. S3A). In contrast, ADAM23 was faintly detected along the axonal surface when co-transfected with Caspr2HA (Fig. S3B). Since Caspr2 is strongly internalized in the somatodendritic compartment (Bel et al., 2009), we analyzed whether ADAM23 could be associated with Caspr2 in endocytic vesicles. Immunoendocytosis of Caspr2 was induced using anti-HA mAb and we observed that, indeed ADAM23 was colocalized with Caspr2 in intracellular vesicles labeled for the early endosome marker EEA1 (Fig. S3C). Next, neurons were co-transfected with TAG-1-GFP or Caspr2-HA together with ADAM23-mCherry to better visualize ADAM23 and perform quantitative analysis. The direct fluorescence of ADAM23-mCherry was strongly detected at the AIS in DIV14 hippocampal neurons, when co-expressed with TAG-1-GFP (Fig. 8A), but not with Caspr2-HA (Fig. 8B). The AIS:axon ratio of ADAM23-mCherry expressed alone or co-transfected with Caspr2 was $1.5 \pm 0.1(n=9)$ and $1.3 \pm 0.1(n=16)$, respectively. ADAM23 was significantly enriched at the AIS when co-transfected with TAG-1, with an AIS:axon ratio of $2.0 \pm 0.2$ $(n=11)$ (Fig. 8C).

Through time-lapse live-cell imaging, we analyzed whether TAG-1, Caspr2 and ADAM23 were sorted within the same axonal transport vesicles (Fig. S4). We observed vesicles colabeled with ADAM23-mCherry and TAG-1-GFP moving along the axon (Fig. S4A-E; Movies 5 and 6). Kymograph analysis of transport events indicated that these vesicles moved in the anterograde and
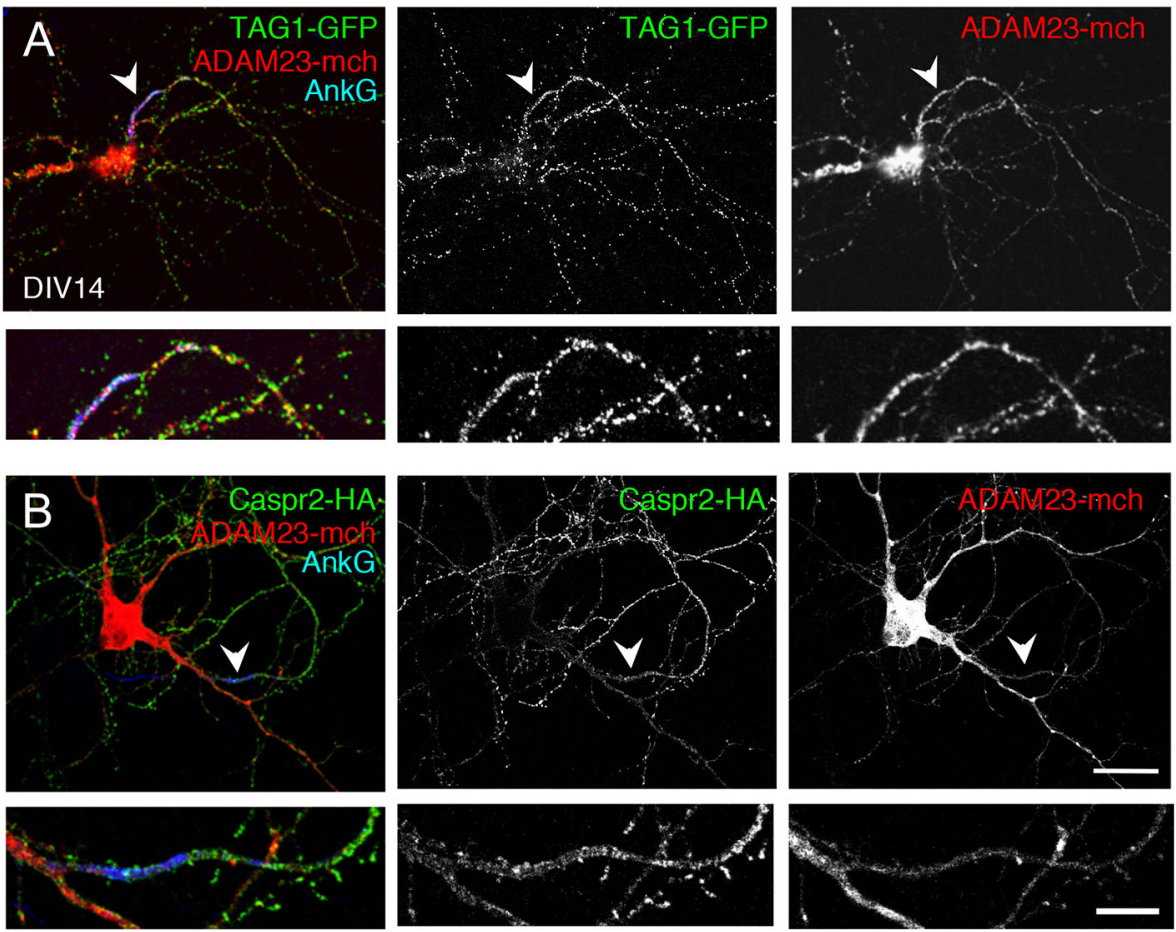

C

AIS/axon

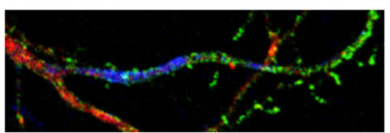

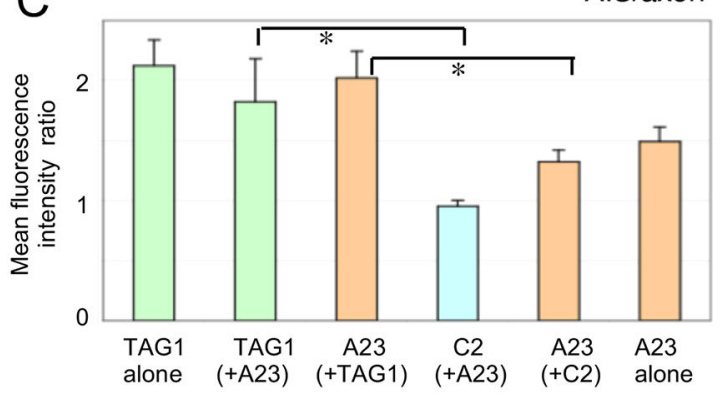

Fig. 8. ADAM23-mCherry is co-targeted with TAG-1-GFP to the AIS of hippocampal neurons. Hippocampal neurons were co-transfected at DIV13 with ADAM23-mCherry and TAG-1-GFP (A), or with Caspr2-HA and ADAM23-mCherry $(B, C)$. Neurons were surface labeled with mouse anti-GFP mAb (A, green) or rat anti-HA (B, green) antibodies and the fluorescence of ADAM23mCherry was directly imaged (red). Cells were fixed and permeabilized before immunostaining for ankyring (blue, arrowheads). Note that ADAM23mCherry was enriched and colocalized with TAG-1 at the AIS (A, magnification) whereas it was faintly detected at the AIS when co-transfected with Caspr2-HA (B). (C) Ratios of mean \pm s.e.m. ( $n=9$ 16) fluorescence intensity between the AIS and axon for TAG-1-GFP, ADAM23-mCherry (A23) and Caspr2 (C2), either transfected alone or cotransfected. ${ }^{*} P<0.01$ (non-parametric MannWhitney test). Scale bars: $20 \mu \mathrm{m}$ (main images); $5 \mu \mathrm{m}$ (magnifications). 
retrograde directions with a $V_{\mathrm{m}}$ of $1.1 \pm 0.3 \mu \mathrm{m} \mathrm{s}^{-1}$ and 0.7 $\pm 0.1 \mu \mathrm{m} \mathrm{s} \mathrm{s}^{-1}$, respectively ( $n=8$ neurons) (Table S2). We determined that vesicles colabeled for TAG-1 and ADAM23 moved mostly retrogradely, as previously observed for TAG-1 (Pinatel et al., 2017). Therefore, ADAM23-mCherry was preferentially transported anterogradely when expressed alone $(65 \pm 9 \%$ of displacements were anterograde; Table S1, Fig. 6B, $\left.\mathrm{B}^{\prime}\right)$. In contrast, it moved mostly retrogradely when associated with TAG-1 (only $34 \pm 6 \%$ of displacements were anterograde; Table S2, Fig. S4B,D). The retrograde vesicles labeled for TAG-1 and ADAM23 are likely endosomes and might provide AIS enrichment at the steady-state by recycling the distal axonal membrane. Next, we examined axonal transport in neurons co-expressing ADAM23-mCherry and Caspr2-GFP (Fig. S4F-H, Movie 7). Kymograph analysis of transport events indicated that these vesicles moved in the anterograde and retrograde directions with a velocity of $1.4 \mu \mathrm{m} \mathrm{s}^{-1}$ and $1 \mu \mathrm{m} \mathrm{s}^{-1}$, respectively (Fig. S4H).

In conclusion, the combinatorial expression of CAMs associated with the Kv1 complex in hippocampal neurons may impact their respective subcellular distribution along the axon. ADAM23 was differentially distributed when co-expressed with TAG-1 or with Caspr2, being either enriched at the AIS or homogenously expressed all along the axon, respectively. Moreover, our data indicate that these CAMs can be sorted together in axonally transported vesicles in cultured hippocampal neurons.

\section{DISCUSSION}

In the present study, we analyzed the axonal targeting of CAMs associated with Kv1 channels in cultured hippocampal neurons. We showed that LGI1 is colocalized with ADAM22 at the AIS of cultured hippocampal neurons. The human mutations LGI1 ${ }^{\text {S473L }}$ and LGI1 ${ }^{\mathrm{R} 474 \mathrm{Q}}$, which are associated with epilepsy, impair the association with ADAM22 and the trapping of LGI1 at the AIS, which is a possible pathogenic mechanism. The co-expression of ADAM22 or ADAM23 strongly modulates the targeting of LGI1, leading to a decrease at the AIS and an increase at the overall neuronal cell surface. We demonstrated that association with ADAM proteins is not only required for the anchoring of LGI1 at the cell surface, but is also implicated in its trafficking, including ER exit, N-glycosylation processing and axonal vesicular transport. In addition, an interplay was evidenced between ADAM proteins and other CAMs of the Kv1 complex, namely, TAG-1 and Caspr2, which may be implicated in their selective targeting at the AIS.

We observed that LGI1 transfected in cultured hippocampal neurons is tethered at the AIS and colocalized with ADAM22 and Kv1 channels. We also found that co-expression of LGI1 with ADAM22 or ADAM23 strongly modulates the subcellular distribution of LGI1. Co-transfection with ADAM proteins strongly increases the surface expression of LGI1 at the somatodendritic and axonal compartments. As a consequence, the enrichment of LGI1 at the AIS becomes reduced in neurons overexpressing ADAM22 and ADAM23. Thus, the association of LGI1 with the Kv1 channels at the AIS, along the axon or at the synaptic terminals may be modulated depending on its association with ADAM22 or ADAM23. ADAM22 is enriched at the AIS of cultured hippocampal neurons and colocalized with Kv1 channels and PSD93, but it is not required for the AIS clustering of Kv1.2 as indicated by the phenotype of Adam $22^{-/-}$mice (Ogawa et al., 2010). However, reciprocally, high expression of ADAM22 or ADAM23 may remove LGI1 associated in a complex with the Kv1 channels from the AIS towards the axonal terminals, through a competition with ADAM22 tethered at the AIS. Indeed, loss-of- function experiments using ADAM22-specific siRNAs indicate that ADAM22 is critical for the trapping of LGI1 at the AIS.

LGI1 has been reported to act presynaptically as a negative regulator of excitatory transmission in early postnatal stages, possibly through Kv1-mediated modulation of synaptic release (Boillot et al., 2016). More recently, LGI1 was shown to localize at the AIS of CA3 hippocampal neurons, regulating action potential firing by controlling the density of Kvl channels (Seagar et al., 2017). LGI1-knockout mice display a downregulation of the expression of Kv1.1 and Kv1.2 via a post-translational mechanism (Seagar et al., 2017), such as altered trafficking or increased degradation, that may contribute to epileptogenesis. Interestingly, here we show that the human missense mutations LGI $1^{\text {S473L }}$ and LGI1 ${ }^{\mathrm{R} 474 \mathrm{Q}}$, which are associated with epilepsy, impair LGI1 trapping at the AIS in hippocampal neurons, which is as a possible pathogenic mechanism. The LGI1 ${ }^{\mathrm{R} 407 \mathrm{C}}$ mutant is properly recruited at the AIS indicating that another function of LGI1 might be affected in this secretion mutant. However, recently, this mutation was found in control individuals indicating that it may not be pathogenic (Yamagata et al., 2018). Furthermore, co-immunoprecipitation experiments indicate that the S473L and R474Q mutations, but not the R407C mutation strongly decrease the interaction with ADAM22 and not with ADAM23. Since ADAM22 is specifically enriched at the AIS of cultured hippocampal neurons, we hypothesize that altered binding with ADAM22 may prevent trapping of LGI1 mutants at the AIS. In a recent report, Yamagata et al. (2018), resolved the crystal structure of the LGI1 in complex with ADAM22 and showed that the R474Q mutation is located at the LGI1-LGI1 interface and does not disrupt the cis-interaction with ADAM22, but does disrupt the higher-order assembly of LGI1-ADAM22 heterotetramers. Thus, homodimerization of LGI1 and its association in complex with ADAM22 may be required for its concentration at the AIS.

The ADAM proteins may act either as chaperones promoting LGI1 export or as anchors to stabilize the secreted LGI1 protein at the neuronal cell surface, which are indeed two non-exclusive processes. First, we demonstrated that LGI1 cell surface targeting is strongly increased by its binding to ADAM22 or ADAM23 both in HEK cells and in neurons. Second, a distinct carbohydrate processing is observed when LGI1 is co-expressed with ADAM22 or ADAM23 in HEK cells with a doublet of Endo H-sensitive and Endo H-resistant glycoforms, whereas an intermediate shift after Endo $\mathrm{H}$ treatment is detected when LGI1 is expressed alone. These results indicate that binding with ADAM proteins may induce a conformational switch further enhancing the ER exit of LGI1. LGI1 may bear distinct carbohydrates when associated or not with ADAMs, or when targeted to the synapses or to the AIS.

In addition, most of the missense mutations identified in ADLTE were classified as secretion-defective mutations, indicating that this genetic disorder could be a conformational disease (Yokoi et al., 2015). The LGI1 ${ }^{\mathrm{S} 473 \mathrm{~L}}$ mutant, which is secreted, was shown to exhibit defective binding activity for ADAM22 in tandem affinity purification using transgenic mouse brain (Yokoi et al., 2015). A consequence of this reduced binding activity might be to interfere with the optimal transport-permissive conformation of the mutant protein. However, we show here that LGI1 ${ }^{\mathrm{S} 473 \mathrm{~L}}$ may sufficiently associate with both ADAM22 and ADAM23 to be properly processed with Endo H-resistant N-glycans in HEK cells. In contrast, this mutation does not allow the molecule to be enriched at the AIS, indicating that LGI1 may require a higher-order association with ADAM22 to be trapped specifically in this axonal subregion. LGI1 is also known to interact with NgR1 (also known as RTN4R), 
which may enhance the association of LGI1 with ADAM22 (Thomas et al., 2016). Whether any mutation in LGI1 linked with epilepsy may induce defects in NgR1 binding is unknown (Thomas et al., 2016). ADAM11 is also known as a ligand for LGI1 (Sagane et al., 2008) that plays a critical role in localizing the Kv1 channels at the presynaptic terminals of cerebellar basket cells (Kole et al., 2015). There is no indication that these ligands of LGI1 are localized at the AIS. Thus, the pathogenic mechanisms for the LGI1 $^{\text {S473L }}$ and LGI1 ${ }^{\text {R474Q }}$ mutations may rely both on defective binding to one of its receptors and to its mistargeting into the different axonal subcompartments, including the AIS and synaptic terminals.

LGI1 has been proposed to form a trans-synaptic bridge through its binding with ADAM22 and ADAM23 expressed at the post- and pre-synapse, respectively. ADAM22 and ADAM23 are partitioned into synaptic fractions depending on LGI1 (Fukata et al., 2010). LGI1 and ADAM22 bind PSD-95 and, consequently, may stabilize the AMPAR and stargazin complex, regulating synaptic strength at the excitatory synapse (Fukata et al., 2006). Interestingly, LGI1 seems to be required for the synaptic localization of ADAM22 and ADAM23 as indicated by immunohistochemical and biochemical analyses of LGI1-deficient mice (Fukata et al., 2010). Conversely, in Adam $22^{-/-}$and Adam $23^{-/-}$mice, the neuropil staining of LGI1 is lower in most hippocampal regions, indicating that the distribution of these molecules at the synaptic neuropil is interdependent (Yokoi et al., 2015). Our results show that LGI1 may associate with ADAM22 or ADAM23 early along the secretory pathway at the ER level to be sorted with either ADAM molecule in axonal transport vesicles. We observed that vesicles containing ADAM22 and ADAM23 either alone or colocalized with LGI1 are axonally transported through the AIS, as reported for axonal cargoes (Al-Bassam et al., 2012; Petersen et al., 2014). The vesicular axonal transport of ADAM23 was predominantly oriented in the anterograde direction, whereas ADAM22 was preferentially transported in the retrograde direction. Such a differential transport may promote a distinct pre- and post-synaptic distribution at the steady state. Therefore, the interdependence of LGI1 and ADAM proteins for their synaptic distribution may be based on their association during axonal transport. As we observed for LGI1 and ADAM proteins, the axonal transport of Kv1.2 subunits associated with the accessory Kv 32 subunits has been reported to occur both in the anterograde and retrograde directions with similar velocities ( $\mathrm{Gu}$ and $\mathrm{Gu}, 2010)$. The axonal vesicular transport of Kv1.2 is facilitated by $\mathrm{Kv} \beta 2$ and depends on the kinesins KIF3A and KIF5B (Gu and Gu, 2010; Rivera et al., 2007). Whether LGI1 and ADAM proteins can traffic together with Kv1 channels as a preformed complex and using identical molecular motors deserves further investigations.

The composition of the Kv1 complex and the mechanisms regulating its recruitment at the AIS are still elusive. The analysis of the Caspr2 interactome in hippocampus indicates that this protein associates with TAG-1 and Kv1, as well as with ADAM22 and LGI1 (Chen et al., 2015). From co-immunoprecipitation experiments of transfected HEK cells, we determined that Caspr2 interacts, through its extracellular or transmembrane domain, with ADAM22 and ADAM23, whereas TAG-1 only precipitated with ADAM22. Moreover, the ADAM proteins can be sorted together with Caspr2 or TAG-1 in axonal transport vesicles. We recently showed that TAG-1 is enriched at the AIS, whereas Caspr2 is uniformly expressed along the axon of cultured hippocampal neurons (Pinatel et al., 2017). Similarly, here we noticed that ADAM23 is enriched at the AIS when co-transfected with TAG-1, but not when co-transfected with Caspr2. Therefore, the ADAM family may play a pivotal role for the interdependent distribution of the different sets of CAMs associated with Kv1 at the AIS. Our results indicate that a focus on AIS might be relevant for the further dissection of the pathogenic mechanisms involving LGI1 in epilepsy.

\section{MATERIALS AND METHODS Constructs}

The pCDNA3-Caspr2-HA construct encodes human Caspr2 with the HA epitope inserted downstream of the signal peptide between the residues Trp26 and Thr27 (Bel et al., 2009). The Caspr2-HA deleted construct Caspr2 $\Delta$ cyt (stop codon at amino acid 1285) was as described previously (Pinatel et al., 2015). NrCAM-GFP was previously described (Falk et al., 2004). The human TAG-1-GFP and Caspr2-GFP constructs with GFP downstream of the signal peptide were as described previously (Pinatel et al., 2015). Caspr2-mCherry was generated by insertion into the EcoRIBamHI sites of pmCherry-N1. Plasmids encoding human LGI1, ADAM22 and ADAM23 were purchased from Origene. LGI1-GFP was generated by insertion in pEGFP-N3 (Pinatel et al., 2015). The R407C, S473L and R474Q missense mutations of LGI1-GFP were generated by using the QuikChange II mutagenesis kit (Stratagene). ADAM22-mCherry and ADAM23-mCherry were generated by insertion into the NheI-KpnI sites of pmCherry-N1. PCR-amplified products were verified by sequencing (Genewiz). Silencer select predesigned siRNAs were purchased from Ambion, Thermo Fisher Scientific. Two different siRNAs targeting rat ADAM22 were used with antisense sequences 5'-UGCGUUGAAAUUCAGAUGGaa-3' in exon 6,7 (ref. s186484) and 5'-UGAGAUUAUACCGAUAUUGtg-3' in exon 12 (ref. s186485). The antisense sequence of the siRNA targeting rat ADAM23 was 5'-AUCGUUAACAAUCAUAAGCtc-3' (ref. s153985) and the negative control siRNA was a universal non-targeting sequence (ref. 4390843).

\section{Western blotting and immunoprecipitation}

HEK cells were co-transfected with Caspr2-HA, Caspr2Acyt-HA, Caspr2GFP, LGI1-GFP, TAG-1-GFP or NrCAM-GFP, and ADAM22-mCherry or ADAM23-mCherry. HEK cells were co-transfected with GFP-tagged LGI1, LGI ${ }^{\mathrm{R} 407 \mathrm{C}}, \mathrm{LGI}^{\mathrm{S} 473 \mathrm{~L}}$ and LGI1 ${ }^{\mathrm{R} 474 \mathrm{Q}}$, and ADAM22-mCherry or ADAM23-mCherry. Cells were lyzed for $30 \mathrm{~min}$ on ice with $50 \mathrm{mM}$ Tris$\mathrm{HCl} \mathrm{pH} \mathrm{7.5,} 1 \% \mathrm{NP}-40,10 \mathrm{mM} \mathrm{MgCl}{ }_{2}$ and protease inhibitors, centrifuged at $4^{\circ} \mathrm{C}$ for $15 \mathrm{~min}$ at $12,000 \mathrm{~g}$. After preclearing for $1 \mathrm{~h}$ at $4^{\circ} \mathrm{C}$ with protein G-Sepharose, supernatants were immunoprecipitated overnight at $4^{\circ} \mathrm{C}$ with protein G-agarose coated with rabbit anti-mCherry (RFP) antibody $(2 \mu \mathrm{l})$ and mouse anti-GFP IgG $(1 \mu \mathrm{g})$ or rat anti-HA $\operatorname{IgG}(1 \mu \mathrm{g})$. The beads were washed twice with $50 \mathrm{mM}$ Tris- $\mathrm{HCl} \mathrm{pH} \mathrm{7.4,150} \mathrm{mM} \mathrm{NaCl}$ and $1 \% \mathrm{NP}-40$, twice in $50 \mathrm{mM}$ Tris- $\mathrm{HCl}, 150 \mathrm{mM} \mathrm{NaCl}$ and twice in $50 \mathrm{mM}$ Tris- $\mathrm{HCl}$. Immunoprecipitates were analyzed by immunoblotting with anti-HA, antiGFP or anti-mCherry antibodies diluted 1:1000. Blots were developed using the ECL chemiluminescent detection system (Roche). HEK cells were transfected with GFP-tagged LGI1 or LGI1 ${ }^{\mathrm{S} 473 \mathrm{~L}}$ alone or co-transfected with ADAM22-mCherry or ADAM23-mCherry. The LGI1 proteins were immunoprecipitated from the lysates using mouse anti-GFP, eluted from protein $\mathrm{G}-\mathrm{Sepharose}$ in $50 \mathrm{mM}$ Tris- $\mathrm{HCl} \mathrm{pH} 6,0.2 \%$ SDS, $1 \%$ $\beta$-mercaptoethanol, $0.5 \%$ Triton X-100, $1 \mathrm{mM}$ EDTA and protease inhibitors and incubated for $3 \mathrm{~h}$ with Endoglycanase $\mathrm{H}(3 \mathrm{mU} / \mu \mathrm{l})$ or

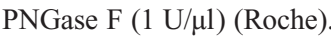

\section{Antibodies and immunofluorescence staining}

The chicken anti-MAP2 (ab5392) and the goat anti-GFP (ab 5450) antibody were purchased from Abcam, the rat anti-HA mAb (clone 3F10) and the mouse anti-GFP (cat. 11814460 001) from Roche, the rabbit anti-RFP (anti-mCherry, code 600-401-379) antiserum from Rockland, the rabbit anti-ADAM23 (cat PA5-30939) and the rabbit anti-synaptobrevin (VAMP2) (cat OSS00035W) from ThermoScientific. The mouse antiankyrinG (clone N106/36), anti-ADAM22 (clone N57/2 and N46/30) antiLGI1 (N283/7), anti-pan neurofascin (clone A12/18) and anti-Kv1.2 (clone K14/16) mAbs were obtained from the UC Davis/NIH NeuroMab facility. 
The mouse anti-TAG-1 1C12 was a gift from Dr Domna Karagogeos (IMBB-FORTH, University of Crete Medical School, Greece) and the chicken anti-ADAM23 from Dr Dies Meijer (Center for Discovery Brain Sciences, University of Edinburgh, UK). Alexa Fluor 488-, 568- and 647-conjugated secondary antibodies were obtained from Molecular Probes. Immunostaining for Caspr2-HA, LGI1-GFP, TAG-1-GFP and ADAM23 (rabbit antibody) was performed on live cells with antibodies diluted 1:500 in culture medium for 30-60 min. Cells were fixed with 4\% paraformaldehyde in PBS for 10 min and permeabilized with $0.1 \%$ Triton $\mathrm{X}-100$ for $10 \mathrm{~min}$. Immunofluorescence staining was performed using chicken anti-MAP2 $(1: 10,000)$, mouse anti-ankyrinG $(1: 100)$, rabbit anti-synaptobrevin (1:500), mouse anti-ADAM22 clone A46/30 (1:100) or chicken anti-ADAM23 (1:1000) antibodies and with secondary antibodies diluted in PBS containing 3\% bovine serum albumin. After washing in PBS, cells were mounted in Mowiol (Calbiochem). Since the direct fluorescence of LGI1-GFP was very low after fixation with paraformaldehyde, the detection of LGI1-GFP at the neuronal surface was performed using live immunolabeling with anti-GFP antibody and either Alexa Fluor 568 or Alexa Fluor 488 secondary antibody.

\section{Cell culture}

Cell culture media and reagents were from Invitrogen. HEK-293 cells recently authenticated and tested for contamination were grown in DMEM containing $10 \%$ fetal calf serum and were transiently transfected using jet PEI (Polyplus transfection, Ozyme). Primary hippocampal cell cultures were prepared from embryonic day 18-Wistar rats. Hippocampi were collected in Hank's balanced salt solution, dissociated with trypsin and plated at a density of $1.210^{5} \mathrm{cells} / \mathrm{cm}^{2}$ on poly-L-lysine coated coverslips. The hippocampal neurons were cultured in Neurobasal supplemented with 2\% B-27, 1\% penicillin-streptomycin and $0.3 \%$ glutamine in a humidified atmosphere containing $5 \% \mathrm{CO} 2$ at $37^{\circ} \mathrm{C}$. Hippocampal neurons were transfected using Lipofectamine 2000 (Thermo Fisher Scientific) with Caspr2-HA Caspr2GFP, TAG-1-GFP, LGI1-GFP, NrCAM-GFP, ADAM22-mCherry and ADAM23-mCherry or co-transfected with two of these constructs at DIV7 or DIV13 and immunostained 1 day later. For siRNA experiments, hippocampal neurons were co-transfected at DIV7 using Lipofectamine 2000 with $1 \mu \mathrm{g}$ LGI1-GFP, $0.5 \mu \mathrm{g}$ mCherry and $30 \mathrm{pM}$ siRNA per $18 \mathrm{~mm}$ coverslips and immunostained 4 days later. All animal experiments were carried out according to the European and Institutional guidelines for the care and use of laboratory animals and approved by the local authority (laboratory's agreement number D13-055-8, Préfecture des Bouches du Rhône).

\section{Confocal microscopy and image analysis}

Image acquisition was performed on a Zeiss laser-scanning microscope LSM780 equipped with a $63 \times 1.32$ NA oil-immersion objective. Images of GFP- or mCherry- or Alexa-Fluor-stained cells were obtained using the $488 \mathrm{~nm}$ band of an argon laser and the $568 \mathrm{~nm}$ and $647 \mathrm{~nm}$ bands of a solidstate laser for excitation. Fluorescence images were collected automatically with an average of two-frame scans. Quantitative image analysis was performed using ImageJ on confocal sections (10-20 neurons in each condition). The fluorescence intensity was measured in two regions of interest (ankyrinG-positive AIS and axon) using identical confocal parameters. Regions corresponding to the AIS were manually selected on ankyrinG images and reported on other channels for intensity measurements. All intensities were corrected for background labeling using the Zen software (Zeiss). Statistical analysis was performed using Statview or GraphPad Prism software. The data was tested for a normal distribution by using a d'Agostino and Pearson's test. For multiple group comparisons, we used one-way ANOVA followed by Fisher's test. The non-parametric Mann-Whitney test was used when the assumption of normality was not possible.

\section{Imaging vesicle transport}

Coverslips with neurons were loaded into a sealed heated chamber in imaging medium (Hank's balanced salt solution, $\mathrm{pH} 7.2$, with $10 \mathrm{mM}$ HEPES and $0.6 \%$ glucose). Recordings were made $18 \mathrm{~h}$ after transfection. The axons were selected on the basis of their much greater length by comparison with dendrites. Live immunostaining using Alexa-Fluor647-conjugated NF186 was performed to visualize the AIS. Vesicle transport was imaged using a Zeiss laser-scanning microscope equipped with a $63 \times 1.32 \mathrm{NA}$ oil-immersion objective and $37^{\circ} \mathrm{C}$ heating chamber. Dual-color recordings were acquired using simultaneous excitation with 488 $\mathrm{nm}(2-4 \%)$ and $561 \mathrm{~nm}$ lasers (1-2\%), and GaSP photomultiplier tube (PMT) 1 for 499-551 and PMT2 for 569-735 detections (562×240 pixels, average 2, open pinhole, $1.5 \mathrm{~s}$ scanning time, streamed time-lapse recording during 3-9 $\mathrm{min}$ ). Kymographs were generated using ImageJ software and contrast inverted so that the fluorescent vesicles correspond to dark lines. Overlapping transport events were analyzed and the velocity measured.

\section{Acknowledgements}

We wish to thank Marie-Pierre Blanchard of the imaging core facility for help with time-lapse recording and image analysis and Aurélie Montheil and Emilie Pallesi of the Platform of Molecular and Cell Biology of INMED for help with siRNA experiments. We are grateful to Michael Seagar, Jérôme Honnorat, Laurence Goutebroze, Christophe Leterrier, Bénédicte Dargent and Francis Castets for helpful discussions and to Valérie Crépel for support and discussions. We thank the University of California Davis/National Institutes of Health NeuroMab Facility.

\section{Competing interests}

The authors declare no competing or financial interests.

\section{Author contributions}

Conceptualization: B.H., C.F.-S.; Investigation: B.H., L.M., K.A., C.F.-S.; Writing original draft: C.F.; Supervision: C.F.-S.; Funding acquisition: C.F.-S.

\section{Funding}

This work was supported by the Association pour la Recherche sur la Sclérose en Plaques (ARSEP) to C.F.-S.

\section{Supplementary information}

Supplementary information available online at

http://jcs.biologists.org/lookup/doi/10.1242/jcs.219774.supplemental

\section{References}

Al-Bassam, S., Xu, M., Wandless, T. J. and Arnold, D. B. (2012). Differential trafficking of transport vesicles contributes to the localization of dendritic proteins. Cell Rep 2, 89-100.

Bel, C., Oguievetskaia, K., Pitaval, C., Goutebroze, L. and Faivre-Sarrailh, C. (2009). Axonal targeting of Caspr2 in hippocampal neurons via selective somatodendritic endocytosis. J. Cell Sci. 122, 3403-3413.

Boillot, M., Lee, C.-Y., Allene, C., Leguern, E., Baulac, S. and Rouach, N. (2016) LGI1 acts presynaptically to regulate excitatory synaptic transmission during early postnatal development. Sci. Rep. 6, 21769

Chen, N., Koopmans, F., Gordon, A., Paliukhovich, I., Klaassen, R. V., van der Schors, R. C., Peles, E., Verhage, M., Smit, A. B. and Li, K. W. (2015). Interaction proteomics of canonical Caspr2 (CNTNAP2) reveals the presence of two Caspr2 isoforms with overlapping interactomes. Biochim. Biophys. Acta 1854 827-833

Davis, J. Q., Lambert, S. and Bennett, V. (1996). Molecular composition of the node of Ranvier: identification of ankyrin-binding cell adhesion molecules neurofascin (mucin+/third FNIII domain-) and NrCAM at nodal axon segments. J. Cell Biol. 135, 1355-1367.

Dazzo, E., Leonardi, E., Belluzzi, E., Malacrida, S., Vitiello, L., Greggio, E. Tosatto, S. C. and Nobile, C. (2016). Secretion-positive LGI1 mutations linked to lateral temporal epilepsy impair binding to ADAM22 and ADAM23 receptors. PLoS Genet. 12, e1006376.

Duflocq, A., Chareyre, F., Giovannini, M., Couraud, F. and Davenne, M. (2011). Characterization of the axon initial segment (AIS) of motor neurons and identification of a para-AIS and a juxtapara-AIS, organized by protein 4.1B. BMC Biol. 9, 66 .

Falk, J., Thoumine, O., Dequidt, C., Choquet, D. and Faivre-Sarrailh, C. (2004) NrCAM coupling to the cytoskeleton depends on multiple protein domains and partitioning into lipid rafts. Mol. Biol. Cell 15, 4695-4709.

Fukata, Y., Adesnik, H., Iwanaga, T., Bredt, D. S., Nicoll, R. A. and Fukata, M. (2006). Epilepsy-related ligand/receptor complex LGI1 and ADAM22 regulate synaptic transmission. Science 313, 1792-1795.

Fukata, Y., Lovero, K. L., Iwanaga, T., Watanabe, A., Yokoi, N., Tabuchi, K., Shigemoto, R., Nicoll, R. A. and Fukata, M. (2010). Disruption of LGI1-linked synaptic complex causes abnormal synaptic transmission and epilepsy. Proc. Natl. Acad. Sci. USA 107, 3799-3804.

$\mathrm{Gu}, \mathrm{Y}$. and Gu, C. (2010). Dynamics of Kv1 channel transport in axons. PLoS ONE 5, e11931.

Gu, W., Brodtkorb, E. and Steinlein, O. K. (2002). LGI1 is mutated in familial temporal lobe epilepsy characterized by aphasic seizures. Ann. Neurol. $\mathbf{5 2}$, 364-367. 
Inda, M. C., DeFelipe, J. and Munoz, A. (2006). Voltage-gated ion channels in the axon initial segment of human cortical pyramidal cells and their relationship with chandelier cells. Proc. Natl. Acad. Sci. USA 103, 2920-2925.

Irani, S. R., Bien, C. G. and Lang, B. (2010). Autoimmune epilepsies. Curr. Opin. Neurol. 24, 146-153.

Kalachikov, S., Evgrafov, O., Ross, B., Winawer, M., Barker-Cummings, C. Martinelli Boneschi, F., Choi, C., Morozov, P., Das, K., Teplitskaya, E. et al. (2002). Mutations in LGI1 cause autosomal-dominant partial epilepsy with auditory features. Nat. Genet. 30, 335-341.

Kegel, L., Aunin, E., Meijer, D. and Bermingham, J. R. (2012). LGI proteins in the nervous system. ASN Neuro 5, 167-181.

Kole, M. H. P. and Stuart, G. J. (2012). Signal processing in the axon initia segment. Neuron 73, 235-247.

Kole, M. J., Qian, J., Waase, M. P., Klassen, T. L., Chen, T. T., Augustine, G. J. and Noebels, J. L. (2015). Selective loss of presynaptic potassium channel clusters at the cerebellar basket cell terminal pinceau in adam11 mutants reveals their role in ephaptic control of purkinje cell firing. J. Neurosci. 35, 11433-11444.

Lai, M., Huijbers, M. G. M., Lancaster, E., Graus, F., Bataller, L., Balice-Gordon, R., Cowell, J. K. and Dalmau, J. (2010). Investigation of LGI1 as the antigen in limbic encephalitis previously attributed to potassium channels: a case series. Lancet Neurol. 9, 776-785.

Lancaster, E., Huijbers, M. G. M., Bar, V., Boronat, A., Wong, A., MartinezHernandez, E., Wilson, C., Jacobs, D., Lai, M., Walker, R. W. et al. (2011) Investigations of caspr2, an autoantigen of encephalitis and neuromyotonia. Ann. Neurol. 69, 303-311

Lasiecka, Z. M., Yap, C. C., Vakulenko, M. and Winckler, B. (2009) Compartmentalizing the neuronal plasma membrane from axon initial segments to synapses. Int. Rev. Cell Mol. Biol. 272, 303-389.

Lovero, K. L., Fukata, Y., Granger, A. J., Fukata, M. and Nicoll, R. A. (2015). The LGI1-ADAM22 protein complex directs synapse maturation through regulation of PSD-95 function. Proc. Natl. Acad. Sci. USA 112, E4129-E4137.

Morante-Redolat, J. M., Gorostidi-Pagola, A., Piquer-Sirerol, S., Saenz, A., Poza, J. J., Galan, J., Gesk, S., Sarafidou, T., Mautner, V. F., Binelli, S. et al. (2002). Mutations in the LGI1/Epitempin gene on 10q24 cause autosomal dominant lateral temporal epilepsy. Hum. Mol. Genet. 11, 1119-1128.

Muona, M., Fukata, Y., Anttonen, A.-K., Laari, A., Palotie, A., Pihko, H. Lönnqvist, T., Valanne, L., Somer, M., Fukata, M. et al. (2016). Dysfunctiona ADAM22 implicated in progressive encephalopathy with cortical atrophy and epilepsy. Neurol Genet 2, e46.

Ogawa, Y., Horresh, I., Trimmer, J. S., Bredt, D. S., Peles, E. and Rasband, M. N (2008). Postsynaptic density-93 clusters Kv1 channels at axon initial segments independently of Caspr2. J. Neurosci. 28, 5731-5739.

Ogawa, Y., Oses-Prieto, J., Kim, M. Y., Horresh, I., Peles, E., Burlingame, A. L., Trimmer, J. S., Meijer, D. and Rasband, M. N. (2010). ADAM22, a Kv1 channelinteracting protein, recruits membrane-associated guanylate kinases to juxtaparanodes of myelinated axons. J. Neurosci. 30, 1038-1048.

Ohkawa, T., Fukata, Y., Yamasaki, M., Miyazaki, T., Yokoi, N., Takashima, H. Watanabe, M., Watanabe, O. and Fukata, M. (2013). Autoantibodies to epilepsyrelated LGI1 in limbic encephalitis neutralize LGI1-ADAM22 interaction and reduce synaptic AMPA receptors. J. Neurosci. 33, 18161-18174.

Owuor, K., Harel, N. Y., Englot, D. J., Hisama, F., Blumenfeld, H. and Strittmatter, S. M. (2009). LGI1-associated epilepsy through altered ADAM23dependent neuronal morphology. Mol. Cell. Neurosci. 42, 448-457.
Petersen, J. D., Kaech, S. and Banker, G. (2014). Selective microtubule-based transport of dendritic membrane proteins arises in concert with axon specification. J. Neurosci. 34, 4135-4147.

Pinatel, D., Hivert, B., Boucraut, J., Saint-Martin, M., Rogemond, V., Zoupi, L., Karagogeos, D., Honnorat, J. and Faivre-Sarrailh, C. (2015). Inhibitory axons are targeted in hippocampal cell culture by anti-Caspr2 autoantibodies associated with limbic encephalitis. Front. Cell Neurosci. 9, 265.

Pinatel, D., Hivert, B., Saint-Martin, M., Noraz, N., Savvaki, M., Karagogeos, D. and Faivre-Sarrailh, C. (2017). The Kv1-associated molecules TAG-1 and Caspr2 are selectively targeted to the axon initial segment in hippocampal neurons. J. Cell Sci. 130, 2209-2220.

Poliak, S., Salomon, D., Elhanany, H., Sabanay, H., Kiernan, B., Pevny, L., Stewart, C. L., Xu, X., Chiu, S.-Y., Shrager, P. et al. (2003). Juxtaparanodal clustering of Shaker-like $\mathrm{K}+$ channels in myelinated axons depends on Caspr2 and TAG-1. J. Cell Biol. 162, 1149-1160.

Rasband, M. N. (2010). The axon initial segment and the maintenance of neuronal polarity. Nat. Rev. Neurosci. 11, 552-562

Rivera, J., Chu, P.-J., Lewis, T. L., Jr. and Arnold, D. B. (2007). The role of Kif5B in axonal localization of Kv1 K(+) channels. Eur. J. Neurosci. 25, 136-146.

Rodenas-Cuadrado, P., Ho, J. and Vernes, S. C. (2014). Shining a light on CNTNAP2: complex functions to complex disorders. Eur. J. Hum. Genet. 22 171-178.

Sagane, K., Ishihama, Y. and Sugimoto, H. (2008). LGI1 and LGI4 bind to ADAM22, ADAM23 and ADAM11. Int. J. Biol. Sci. 4, 387-396.

Sánchez-Ponce, D., DeFelipe, J., Garrido, J. J. and Muñoz, A. (2012) Developmental expression of $\mathrm{Kv}$ potassium channels at the axon initial segment of cultured hippocampal neurons. PLoS ONE 7, e48557.

Seagar, M., Russier, M., Caillard, O., Maulet, Y., Fronzaroli-Molinieres, L., De San Feliciano, M., Boumedine-Guignon, N., Rodriguez, L., Zbili, M., Usseglio, F. et al. (2017). LGI1 tunes intrinsic excitability by regulating the density of axonal Kv1 channels. Proc. Natl. Acad. Sci. USA 114, 7719-7724.

Thomas, R. A., Ambalavanan, A., Rouleau, G. A. and Barker, P. A. (2016). Identification of genetic variants of LGI1 and RTN4R (NgR1) linked to schizophrenia that are defective in NgR1-LGI1 signaling. Mol. Genet. Genomic Med. 4, 447-456

Traka, M., Goutebroze, L., Denisenko, N., Bessa, M., Nifli, A., Havaki, S. Iwakura, Y., Fukamauchi, F., Watanabe, K., Soliven, B. et al. (2003) Association of TAG-1 with Caspr2 is essential for the molecular organization of juxtaparanodal regions of myelinated fibers. J. Cell Biol. 162, 1161-1172.

Vacher, H. and Trimmer, J. S. (2012). Trafficking mechanisms underlying neuronal voltage-gated ion channel localization at the axon initial segment. Epilepsia 9, 21-31.

Vacher, H., Yang, J.-W., Cerda, O., Autillo-Touati, A., Dargent, B. and Trimmer, J. S. (2011). Cdk-mediated phosphorylation of the Kvbeta2 auxiliary subunit regulates Kv1 channel axonal targeting. J. Cell Biol. 192, 813-824.

Yamagata, A., Miyazaki, Y., Yokoi, N., Shigematsu, H., Sato, Y., Goto-Ito, S., Maeda, A., Goto, T., Sanbo, M., Hirabayashi, M. et al. (2018). Structural basis of epilepsy-related ligand-receptor complex LGI1-ADAM22. Nat. Commun. 9, 1546

Yokoi, N., Fukata, Y., Kase, D., Miyazaki, T., Jaegle, M., Ohkawa, T., Takahashi, N., Iwanari, H., Mochizuki, Y., Hamakubo, T. et al. (2015). Chemical correcto treatment ameliorates increased seizure susceptibility in a mouse model of familial epilepsy. Nat. Med. 21, 19.

Zhang, C. and Rasband, M. N. (2016). Cytoskeletal control of axon domain assembly and function. Curr. Opin. Neurobiol. 39, 116-121. 